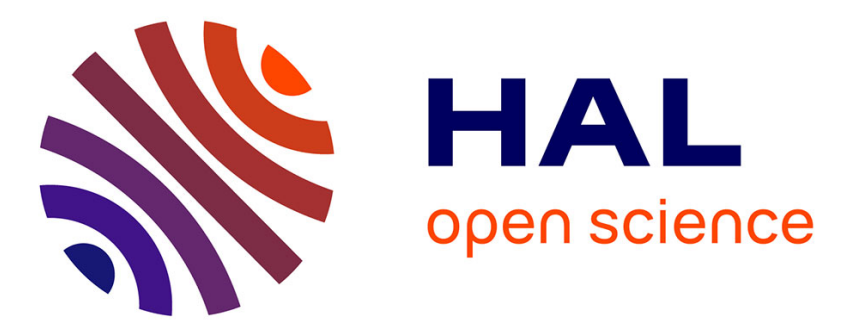

\title{
Stress- and pathway-specific impacts of impaired jasmonoyl-isoleucine (JA-Ile) catabolism on defense signaling and biotic stress resistance
}

Valentin Marquis, Ekaterina Smirnova, Laure Poirier, Julie Zumsteg, Fabian Schweizer, Philippe Reymond, Thierry Heitz

\section{To cite this version:}

Valentin Marquis, Ekaterina Smirnova, Laure Poirier, Julie Zumsteg, Fabian Schweizer, et al.. Stress- and pathway-specific impacts of impaired jasmonoyl-isoleucine (JA-Ile) catabolism on defense signaling and biotic stress resistance. Plant, Cell and Environment, 2020, 43, pp.1558 - 1570. $10.1111 /$ pce. 13753 . hal-02613222

\section{HAL Id: hal-02613222 \\ https://hal.science/hal-02613222}

Submitted on 3 Dec 2020

HAL is a multi-disciplinary open access archive for the deposit and dissemination of scientific research documents, whether they are published or not. The documents may come from teaching and research institutions in France or abroad, or from public or private research centers.
L'archive ouverte pluridisciplinaire HAL, est destinée au dépôt et à la diffusion de documents scientifiques de niveau recherche, publiés ou non, émanant des établissements d'enseignement et de recherche français ou étrangers, des laboratoires publics ou privés. 
Stress- and pathway-specific impacts of impaired jasmonoyl-isoleucine (JA-Ile)

catabolism on defense signaling and biotic stress resistance

Valentin Marquis $^{1} \dagger$, Ekaterina Smirnova ${ }^{1} \dagger$, Laure Poirier ${ }^{1}$, Julie Zumsteg ${ }^{1}$, Fabian

Schweizer ${ }^{2}$, Philippe Reymond ${ }^{2}$ and Thierry Heitz ${ }^{1 *}$

1 Institut de Biologie Moléculaire des Plantes (IBMP) du CNRS, Université de Strasbourg, Strasbourg, France

2 Department of Plant Molecular Biology, University of Lausanne, Lausanne, Switzerland

† These authors contributed equally to the work

\section{*Correspondance}

Thierry Heitz, IBMP-CNRS, Université de Strasbourg, Strasbourg, France

E-mail : thierry.heitz@ibmp-cnrs.unistra.fr

Running head: Pathway-specific impact of JA-Ile catabolism on defense

\section{Funding information}

CNRS, grants ANR-12-BSV8-005 from the Agence Nationale de la Recherche and IdEx2014-208e Interdisciplinary grant from Université de Strasbourg and CNRS, Université de differences between this version and the Version of Record. Please cite this article as doi: $10.1111 /$ pce. 13753 
Strasbourg and the Ministère de l'Enseignement Supérieur et de la Recherche to T.H. Swiss National Science Foundation grant number 31003A_169278 to P.R.

\section{Abstract}

Jasmonate synthesis and signaling are essential for plant defense upregulation upon herbivore or microbial attacks. Stress-induced accumulation of jasmonoyl-isoleucine (JA-Ile), the bioactive hormonal form triggering transcriptional changes, is dynamic and transient, due to the existence of potent removal mechanisms. Two JA-Ile turnover pathways operate in Arabidopsis, consisting in cytochrome P450 (CYP94)-mediated oxidation and deconjugation by the amidohydrolases (AH) IAR3/ILL6. Understanding their impacts was previously blurred by gene redundancy and compensation mechanisms. Here we address the consequences of blocking these pathways on jasmonate homeostasis and defenses in double2ah, triple-3cyp mutants, and a quintuple-5ko line deficient in all known JA-Ile-degrading activities. These lines reacted differently to either mechanical wounding/insect attack or fungal infection. Both pathways contributed additively to JA-Ile removal upon wounding, but their impairement had opposite impacts on insect larvae feeding. In contrast, only the $a h$ pathway was essential for JA-Ile turnover upon infection by Botrytis, yet only 3cyp was more fungus-resistant. Despite of building-up extreme JA-Ile levels, 5ko displayed near-wild-type resistance in both bioassays. Molecular analysis indicated that restrained JA-Ile catabolism resulted in enhanced defense/resistance only when genes encoding negative regulators were 
not simultaneously overstimulated. This occurred in discrete stress- and pathway-specific combinations, providing a framework for future defense-enhancing strategies.

\section{Keywords:}

Arabidopsis, Botrytis, catabolism, defense, feedback, jasmonate, resistance, signaling, wounding

\section{Acknowledgements:}

We thank J. Browse (WSU, Pullman, USA) for providing a segregating population of iar3-5 ill6-2 line.

\section{Summary statement}

We investigated the impacts of impairing catabolic pathways of the defense hormone jasmonoyl-isoleucine in higher order mutants of Arabidopsis thaliana. The data disclosed pathway-specific changes on resistance to herbivory or fungal infection, that correlated with feedback inhibition responses.

\section{INTRODUCTION}


Jasmonates (JAs) have been recognized for more than two decades as powerful regulators of inducible defense responses protecting plants from damage inflicted by herbivorous insects or microbial pathogens (Campos, Kang, \& Howe, 2014; Wasternack \& Hause, 2013). Even before the identification of jasmonoyl-(L)-isoleucine (JA-Ile) as the critical bioactive form in 2007 (Chini et al., 2007; Thines et al., 2007), many derivatives of jasmonic acid (JA) were described, resulting from hydroxylation, conjugation to sugars (Miersch, Neumerkel, Dippe, Stenzel, \& Wasternack, 2008), amino acids or amino cyclopropane carboxylic acid (Staswick \& Tiryaki, 2004), sulfation (Gidda et al., 2003), decarboxylation and many more (Wasternack \& Song, 2017). The enzymes responsible for these modifications have not all been identified and the functions of these derivatives, if any, are generally unknown. The master regulator JA-Ile results from a specific conjugation reaction of jasmonic acid (JA) by the enzyme JASMONATE RESISTANT 1 (JAR1) (Staswick \& Tiryaki, 2004). In the core perception and signaling pathway, JA-Ile acts as a ligand promoting the assembly of the co-receptor CORONATINE INSENSITIVE 1 (COI1) with distinct JASMONATE ZIM-DOMAIN PROTEIN (JAZ) that otherwise powerfully repress target transcription factors (TF) and their cognate responses. After its formation, the COI1-JA-Ile-JAZ complex is recruited into the E3 ubiquitin ligase $\mathrm{SCF}^{\mathrm{COI} 1}$ that tags JAZs for proteolytic degradation, and provides the basis for JA-Ile-responsive gene de-repression (Chini et al., 2007; Thines et al., 2007). Several hundreds to thousands of genes are under this control, and depending on developmental stage, organ, nature of stimulus, and crosstalks with other hormones, the existence of a wide array of JAZ-TF combinations provides specificity to the system with only one major hormonal ligand 
(Chini, Gimenez-Ibanez, Goossens, \& Solano, 2016). Distinct JA-Ile-triggered networks regulate leaf defenses against various aggressors and are integrated by separate sets of TFs. MYC2, a bHLH type TF, integrates (together with MYC3 and MYC4) simultaneous JA/abscisic acid (ABA) stimuli and defines a wound/insect-specific branch including the induction of typical responses such as vegetative storage proteins (VSPs) or glucosinolates (Fernandez-Calvo et al., 2011; Schweizer et al., 2013); ERF1/ORA59 TFs integrate concomitant JA/ethylene (ET) signals into a microbe-specific branch controling expression of plant defensins, typically PDF1.2 (Pieterse, Van der Does, Zamioudis, Leon-Reyes, \& Van Wees, 2012; Wasternack \& Hause, 2013).

JA signaling, like any hormonal pathway, needs to be tightly controlled in time and space, particularly because defense upregulation is costly and connected to overall growth inhibition (Guo, Major, \& Howe, 2018). This antagonism was long thought to be merely imposed by limited resources that need to be re-allocated from developmental to defensive sinks (Havko et al., 2016), but recent advances have evidenced hardwire connections in the control of growth-defense tradeoffs (Campos et al., 2014; Guo et al., 2018). For appropriate control of JA responses, plants rely on several negative feedback mechanisms. In addition to JAZ repressor proteins, other negative regulators were identified that repress JA responses at the level of gene promoters, including JAV1 (Yan et al., 2018), and the JAM subclass of bHLH proteins that compete with MYC2/3/4 TFs (Sasaki-Sekimoto et al., 2013). Another way to repress or terminate jasmonate action is at the metabolic level, either by diverting the flux to hydroxy-JA rather than to JA-Ile (Smirnova et al., 2017), or by modifying JA-Ile to alleviate its receptor-binding properties. The characterization of JA-Ile catabolic pathways has 
shed light on hormone homeostasis regulation and has also provided a framework explaining additional complexity in the JA metabolic grid (Heitz et al., 2016). Two distinct enzymatic pathways are known to modify or degrade JA-Ile. One consists in a two-step oxidation of the terminal $(\omega)$ carbon of the JA moiety by cytochrome P450 monooxygenases of the CYP94 family. In Arabidopsis, CYP94B3 and to a minor extent CYP94B1 generate 12OH-JA-Ile as a main product whereas CYP94C1 catalyzes also the full oxidation of JA-Ile to 12COOH-JAIle (Figure 1) (Bruckhoff et al., 2016; Heitz et al., 2012; Kitaoka et al., 2011; Koo, Cooke, \& Howe, 2011; Koo et al., 2014). The first oxidation product retains weak receptor-assembly capacity and gene-inducing activity (Aubert, Widemann, Miesch, Pinot, \& Heitz, 2015; Koo et al., 2011; Poudel et al., 2019), but the second product proved fully inactive (Aubert et al., 2015; Koo et al., 2014). The second pathway is a deconjugation of JA-Ile by an amidohydrolase initially reported in Nicotiana attenuata (Woldemariam, Onkokesung, Baldwin, \& Galis, 2012) of which the Arabidopsis orthologs IAR3 and ILL6 (Figure 1) (Widemann et al., 2013). These enzymes act on JA-Ile and other JA-amino acid conjugates but also on 12OH-JA-Ile generated by CYP94Bs, releasing JA and 12OH-JA, respectively (Widemann et al., 2013; Zhang et al., 2016). The interconnection of the two catabolic pathways generates increased metabolic complexity because many of the above-mentioned JAs derive in fact from JA-Ile catabolism (Heitz et al., 2016; Widemann et al., 2013). Expression of both JA-Ile oxidases and amidohydrolases genes is induced by environmental cues and co-regulated with the central regulon defined by JA pathway biosynthetic and signaling genes. Their simultaneous enzymatic action shapes specific jasmonate patterns in different tissues and stress conditions (Aubert et al., 2015; Heitz et al., 2012; Koo \& Howe, 
2012; Koo et al., 2014; Widemann et al., 2013; Widemann, Smirnova, Aubert, Miesch, \& Heitz, 2016).

Manipulating JA-Ile catabolic routes could be an attractive tool to engineer plants for enhanced pathogen resistance or modulate other JA-dependent processes. Loss- or gain-offunction mutant lines in CYP94 or $A H$ genes have revealed profound impacts on JA metabolism but contrasted consequences on JA responses. Arabidopsis lines ectopically overexpressing CYP94 or $A H$ generally have reduced JA-Ile levels and a metabolic shift towards oxidized and/or cleaved derivatives. As expected, increased turnover is correlated with attenuated JA responses, for example increased sensitivity to Botrytis infection or to insect larvae feeding (Aubert et al., 2015; Koo et al., 2011; Zhang et al., 2016). In comparison, analysis of single, double, or triple knock out mutant lines has not led to a unified conclusion as to how JA-Ile responses are impacted by gradual impairement of JA-Ile catabolism. Deficiency in CYP94 or AH expression impacts JA homeostasis consistently with their in vitro enzymatic activities. For example, cyp94b3 or b3c1 mutations lead to more JAIle accumulation upon leaf wounding, concomitantly to reduced 12OH-JA-Ile and 12COOHJA-Ile levels (Heitz et al., 2012; Kitaoka et al., 2011; Koo et al., 2011). Double cyp94b3c1 and triple cyp94b1b3c1 mutant plants displayed slightly enhanced defense responses and tolerance to fungal infection (Aubert et al., 2015). Strikingly, in another study, the double cyp94b1b3 and triple cyp94b1b3c1 mutants exhibited deficient JA responses, questioning the current signaling model (Poudel et al., 2016). On the other hand, AH-deficient lines have not yet been tested for defense and resistance responses, so the contribution and impacts of this pathway are unknown. 
To overcome gene redundancy and compensation mechanisms between pathways that occur frequently in JA metabolic mutants (Smirnova et al., 2017; Widemann et al., 2013), we introduced in the present study new plant lines with higher order mutations (Figure 1): a double iar3 ill6 mutant (thereafter called 2ah) impaired in the JA-Ile deconjugating pathway; and a quintuple cyp94b1 cyp94b3 cyp94c1 iar3 ill6 (thereafter called 5ko) deficient in all characterized enzymes turning over JA-Ile. We submitted these lines, along with the oxidation-deficient cyp94b1b3c1 mutant (thereafter called 3cyp), to parallel leaf stimulation by mechanical wounding or B. cinerea infection, two environmental cues triggering strong JA metabolism and signaling, but activating distinct transcriptional networks due to different cross-talks with other hormonal pathways (Pieterse et al., 2012). We determined the contribution of each catabolic pathway on JA-Ile turnover for each stress and investigated the impact of modified JA-Ile homeostasis on defense responses and tolerance to aggression by an herbivore insect or by fungal infection (Figure 1). The data highlight new stress-specific impacts of fully impairing either one or both JA-Ile catabolic pathways, where defense and resistance phenotypes are more reflecting the transcriptional behavior of negative feedback signaling effectors than actual JA-Ile levels.

\section{MATERIALS AND METHODS}

\subsection{Plant growth and treatments}

Arabidopsis thaliana genotypes used were in the Col-0 ecotype and were grown under a $12 \mathrm{~h}$ 
light/12 h dark photoperiod in a growth chamber. The individual T-DNA insertion lines were obtained from the Nottingham Arabidopsis Stock Center (NASC). The 3cyp line was obtained by crossing the alleles cyp94b1-1 (SALK_129672), cyp94b3-1 (CS302217) and cyp94c1-1 (SALK_55455) (Aubert et al., 2015). The 2ah line was obtained after crossing the lines iar3-5 (SALK_069047) and ill6-2 (SALK_024894C). The quintuple 5ko line was obtained by crossing the 3cyp line with a double iar3-5 ill6-1 (GK412-E11).

B. cinerea inoculation and resistance scoring were as described in Aubert et al. (2015). For mechanical wounding experiments, 5 or 6 fully expanded leaves were wounded three times across mid-vein with a hemostat. At increasing time points following damage, leaf samples were quickly harvested and flash-frozen in liquid nitrogen before storing at $-80^{\circ} \mathrm{C}$ until analysis.

Exogenous hormone feeding experiments were performed on seedlings as described in Smirnova et al. (2017). Briefly, surface-sterilized seeds were germinated in 24-well microtiter plates under a $16 \mathrm{~h}$ light/ $8 \mathrm{~h}$ dark photoperiod at $22^{\circ} \mathrm{C}$ in a controlled growth chamber in the following medium: 1 x Murashige and Skoog liquid medium, supplemented with 0.5\% sucrose and $0.05 \%$ MES buffer. Seedlings were grown for 7 days before gentle addition of hormones (amino-cyclopropane carboxylic acid, ACC, Sigma-Aldrich A3903) or abscisic acid (ABA, Olchemim 0132721) from stock solutions to a final concentration of $15 \mu \mathrm{M}$. JAIle was chemically synthesized as described in Wiedemann et al. (2013). At increasing time points after treatment, seedlings were quickly harvested and flash-frozen in liquid nitrogen before storing at $-80^{\circ} \mathrm{C}$ until RNA extraction. 


\subsection{Insect performance assay}

Four-week-old Arabidopsis plants were challenged with freshly hatched Spodoptera littoralis larvae (eggs obtained from Syngenta, Stein AG, Switzerland). Five larvae were placed on each of 11 pots, each containing 2 plants. Plants were placed in a transparent plastic box and kept in a growth chamber during the experiment $\left(10 \mathrm{~h}\right.$ light $/ 14 \mathrm{~h}$ dark, $100 \mu \mathrm{mol} \mathrm{m}{ }^{-2} \mathrm{~s}^{-1}$ of light, $20-22^{\circ} \mathrm{C}$ and $65 \%$ relative humidity). After 8-9 days of feeding, larvae were weighed on a precision balance (Mettler-Toledo, Greifensee, Switzerland). The experiment was performed successively three times (different sampling dates).

\subsection{RT-qPCR gene expression assays}

Total RNA was extracted from plant leaves with TRI reagent (Molecular Research Center). One microgram of RNA was reverse transcribed using the SuperScript IV reverse transcription system (Thermo Fisher Scientific). Real-time PCR was performed on 10 ng of cDNA as described in Berr et al. (2010) using a LightCycler 480 II instrument (Roche Applied Science). The housekeeping genes EXP (At4g26410) and TIP41 (At4g34270) were used as internal references for qPCR on cDNA derived from infected/wounded or nonstimulated leaves. Measurement of fungal biomass was performed as described in Smirnova et al., (2017) except that ACT2 (At3g18780) and UBQ10 (At4g05320) were used as reference genes. Gene-specific primer sequences used for qPCR are listed in Table S1.

\subsection{Jasmonate and camalexin profiling}


Jasmonates were identified and quantified by ultra high performance liquid chromatography coupled to tandem mass spectrometry (UHPLC-MS/MS). About 50-100 mg frozen plant material was extracted with 10 volumes $(10 \mu \mathrm{L}$ per $\mathrm{mg})$ of ice-cold extraction solution (MeOH:water:acetic acid 70:29:0.5, v/v/v) containing 9,10-dihydro-JA and 9,10-dihydro-JAIle as internal standards for workup recovery. Grinding was performed with a glass-bead Precellys tissue homogenizer (Bertin Instruments, France) in $2 \mathrm{~mL}$ screw-capped tubes. After 30 min incubation at $4^{\circ} \mathrm{C}$ on a rotating wheel, homogenates were cleared by centrifugation before concentration of supernatants under a stream of $\mathrm{N}_{2}$ and overnight conservation at $20^{\circ} \mathrm{C}$. After a second centrifugation step, extracts were submitted to quantitative LC-MS/MS analysis on an EvoQ Elite LC-TQ (Bruker) equiped with an electrospray ionisation source (ESI) and coupled to a Dionex UltiMate 3000 UHPLC system (Thermo). Five $\mu \mathrm{L}$ plant extract were injected. Chromatographic separation was achieved using an Acquity UPLC HSS T3 column (100 x $2.1 \mathrm{~mm}, 1.8 \mu \mathrm{m}$; Waters) and pre-column. The mobile phase consisted of (A) water and (B) methanol, both containing $0.1 \%$ formic acid. The run started by 2 min of $95 \% \mathrm{~A}$, then a linear gradient was applied to reach $100 \% \mathrm{~B}$ at $10 \mathrm{~min}$, followed by isocratic run using B during 3 min. Return to initial conditions was achieved in 1 min, with a total run time of $15 \mathrm{~min}$. The column was operated at $35^{\circ} \mathrm{C}$ with a flow-rate of $0.30 \mathrm{~mL}$ $\min ^{-1}$. Nitrogen was used as the drying and nebulizing gas. The nebulizer gas flow was set to $35 \mathrm{~L} \mathrm{~h}^{-1}$, and the desolvation gas flow to $30 \mathrm{~L} \mathrm{~h}^{-1}$. The interface temperature was set to $350{ }^{\circ} \mathrm{C}$ and the source temperature to $300^{\circ} \mathrm{C}$. The capillary voltage was set to $3.5 \mathrm{kV}$, the ionization was in positive or negative mode. Low mass and high mass resolution were 2 for the first 
mass analyzer and 2 for the second. Data acquisition was performed with the MS Workstation 8 for the mass spectrometry and the liquid chromatography was piloted with Bruker Compass Hystar 4.1 SR1 software. The data analysis was performed with the MS Data Review software. Absolute quantifications were achieved by comparison of sample signal with doseresponse curves established with pure compounds and recovery correction based on internal standard signal. The transitions were, in negative mode: JA 209.3>59.3; JA-Ile 322.3>130.2; 12OH-JA-Ile 338.3>130.2; 12COOH-JA-Ile 352.2>130.1; 12OH-JA 225.2>59.3; in positive mode: camalexin 201.0>59.3.

\subsection{Statistical analysis}

All statistical analysis was performed using InfoStat 2015d (http:// www.infostat.com.ar). Comparisons of sample means were performed by one-way analysis of variance $(P \% 0.05$ or $P \% 0.01)$ and Tukey's post-hoc multiple comparisons tests $(P<0.05$ or $P<0.01)$, and significant differences of means were determined.

\section{RESULTS}

3.1 Jasmonate profiling of higher order catabolic mutants reveals stimulus-specific impacts on hormone homeostasis 
Effective inactivation of the respective genes was verified in each mutant line by RT-qPCR using RNA extracted from 1 h-wounded leaves (Figure S1 and Aubert et al., 2015)). The four plant genotypes in the Col-0 ecotype (wild-type WT, 2ah, 3cyp, 5ko) were submitted separately to mechanical wounding or to $B$. cinerea inoculation, to activate JA metabolism and responses. Mechanical wounding constitutes a synchronous and severe stimulus, and generates a massive jasmonate pulse where compound-specific dynamics can be followed (Chung et al., 2008; Glauser et al., 2008; Heitz et al., 2012). Therefore, a kinetic study was conducted with tissue collected at 1, 3 and 6 h post-wounding (hpw). In contrast, necrotic lesions inflicted by $B$. cinerea infection develop radially with fungal hyphae continuously infecting new tissue (an asynchronous stimulus), and 3 days post-inoculation (dpi) constitutes an optimal time point for recording biochemical changes and for assessing antifungal resistance. We quantified levels of JA, 12OH-JA, JA-Ile and its catabolites 12OH-JA-Ile and 12COOH-JA-Ile in the two biological responses. JA was less accumulated in infected 3cyp (Figure S2a,b). Its oxidation product, 12OH-JA, is known to be partially formed via conjugate intermediates (Figure 1) upon wounding (Widemann et al., 2013) and accordingly, was less abundant in all mutants at 3 hpw, remaining low in 2ah at 6 hpw (Figure S2). In contrast, $12 \mathrm{OH}-\mathrm{JA}$ was less affected by the mutations upon infection and was even enhanced in $5 \mathrm{ko}$, in accordance with the predominant contribution of Jasmonic Acid Oxidase (JAO) enzymes directly oxidizing JA in response to Botrytis (Figure 1) (Smirnova et al., 2017).

\subsubsection{Impacts on JA-Ile homeostasis upon wounding}


We next examined the evolution of JA-Ile and its oxidized catabolites. JA-Ile profiles showed the typical pattern (Chung et al., 2008; Glauser et al., 2008; Heitz et al., 2012; Koo et al., 2011) in wounded WT leaves, peaking at 1 hpw and declining thereafter (Figure 2a). In 2ah and 3cyp lines, a significant overaccumulation of JA-Ile was recorded at $1 \mathrm{hpw}$ and was prolonged in 3cyp, extending data from lower order cyp94 mutants described previously (Heitz et al., 2012; Widemann et al., 2013; Zhang et al., 2016). In the $5 k$ line that has both pathways impaired, a huge JA-Ile accumulation was recorded that culminated between 3-6 hpw close to 40-50 nmol g FW-1. These data indicate that in WT wounded leaves, both AH and CYP94 pathways contribute similarly to JA-Ile turnover and that their simultaneous inactivation synergistically boosts hormone hyperaccumulation at later time points. Profiles of CYP94-generated JA-Ile oxidation products were as expected: 12OH-JA-Ile levels were about double in 2ah compared to WT, were suppressed in 3cyp and of note, were higher in $5 k o$ than in 3cyp at 6 hpw (Figure 2a). 12COOH-JA-Ile was only detected from 3 hpw and evolved similarly to $12 \mathrm{OH}-\mathrm{JA}-\mathrm{Ile}$ in $2 a \mathrm{~h}$ and 3cyp, but was barely detected in $5 \mathrm{ko}$.

\subsubsection{Impacts on JA-Ile homeostasis upon Botrytis infection}

The metabolic impacts of mutations were different in response to B. cinerea inoculation. JAIle levels were similar to WT in 3cyp, confirming our previous data (Aubert et al., 2015) (Figure 2b). In contrast, steady-state JA-Ile levels were strongly enhanced in $2 a h$ but no further increase was observed in $5 k o$. This result indicates that the amidohydrolase pathway is essential for JA-Ile clearance upon fungal infection and that blocking simultaneously the 
oxidative pathway does only marginally impact JA-Ile accumulation. 2ah also considerably enhanced 12OH-JA-Ile and 12COOH-JA-Ile levels, reinforcing initial trend obtained with iar3 or ill6 single mutants (Widemann et al., 2013). In 2ah, excess uncleaved JA-Ile is likely oxidized to 12OH-JA-Ile that can no more be deconjugated by IAR3 and ILL6 and part of this enlarged pool is further oxidized to 12COOH-JA-Ile by CYP94C1. Together, these experiments demonstrate stimulus-specific contributions of AH and CYP94 catabolic pathways to JA-Ile turnover and accumulation of downstream derivatives.

\subsection{Impact of impaired catabolic pathways on defense and resistance responses is not reflecting JA-Ile accumulation in mutant plants}

As the mutations altered the steady-state levels of bioactive JA-Ile, the lines were examined for induced expression of typical JA-regulated marker genes for each leaf stress model. In the case of wounding, transcripts of MYC2, an early-responsive TF gene controlling late targets, accumulated similarly at $1 \mathrm{hpw}$, but declined less than WT in $2 a h$ and $5 k o$ mutants as best visible at 6 hpw (Figure 3a). Two VSP genes were examined as late markers of the anti-insect branch of the JA defense pathway (Pieterse et al., 2012; Wasternack \& Hause, 2013). In WT, both VSP1 and VSP2 expression peaked at 3 hpw before declining to low levels at 6 hpw (Figure 3a). Expression was globally enhanced in mutant lines compared to WT, but with distinct patterns. VSP1 was strongly enhanced in $2 a h$ at all time points, while in 3cyp and 5ko, persistence in transcript levels was only observed at 6 hpw when WT signal faded. For VSP2, only $2 a h$ displayed enhanced expression at all 3 time points. This indicates that impaired JA- 
Ile catabolism results in persistent defense marker expression, but this is not commensurate to JA-Ile accumulation (Figure 2a), because $5 k o$ displays lower defense than $2 a h$. To determine the physiological impacts of such altered hormone and defense profiles, we used an insect feeding assay as a biological readout of signaling. When larvae of the generalist herbivore Spodoptera littoralis were placed on leaves of the four genotypes for 8 to 9 days, contrasted results were recorded: larvae fed on $2 a h$ plants were consistently lighter than those feeding on WT, indicating stronger anti-insect resistance (Figure 3b). This opposed to 3cyp, that sustained higher insect development, confirming impaired resistance reported previously by Poudel et al. (2016). Surprisingly, 5ko displayed WT level of resistance to herbivory. These conclusions were drawn from 3 successive trials (Figure S3) with large insect populations.

A similar defense-resistance analysis was conducted for the response to $B$. cinerea infection. The antimicrobial branch of JA-Ile-dependent defense signaling was probed by monitoring ORA59 TF and PDF1.2 defense marker expression. Unexpectedly, both genes exhibited reduced expression in $2 a h$ and $5 k o$ at $3 \mathrm{dpi}$, in contrast to 3cyp that maintained WT transcript levels (Figure 4a). Anti-fungal resistance assay indicated that only 3cyp displayed smaller lesions, in agreement with our previous report (Aubert et al., 2015), while 3cyp and to a lesser extent $2 a h$ supported reduced fungal biomass as estimated from $B$. cinerea cutinase signal (Figure 4b). Of note, 5ko behaved essentially like WT in these infection assays. Contents in camalexin, a major antimicrobial phytoalexin in Arabidopsis, were determined but no significant differences were found between genotypes. Together, the data show that impairing either one or both JA-Ile catabolic pathways has distinct and stress-specific consequences on JA-mediated defense and resistance responses. 


\subsection{Impaired JA-Ile catabolism results in gain in resistance only when negative feedback effectors are not overinduced}

Upon parallel investigation of the two leaf defense models, increased JA-Ile accumulation due to impaired catabolism did not correlate systematically with stronger defense or resistance phenotypes. Conversely, enhanced antifungal resistance occured in 3cyp without elevated hormone levels (Figures 2 and 4). These observations suggest that additional players may be at work to limit or counteract overinduction of defense responses under deficient JA-Ile turnover. Obvious candidates for such negative regulation are genes encoding JAZ repressors (Browse, 2009) or JAM bHLH factors competing with MYC TFs (Sasaki-Sekimoto et al., 2013), or JAV1 repressor (Hu et al., 2013), all classes being JA-Ile- and stress-responsive. We selected $J A Z$ genes that were previously described as highly induced and sensitive to catabolic pathway deregulation (Aubert et al., 2015; Heitz et al., 2012; Widemann et al., 2013). As shown in Figure 5a, JAZ8, JAZ10, JAM1, JAM2 and JAV1 expression was maximal in WT at 1 hpw with only slight enhancement in some mutants, but their transcripts were clearly more persistent in 3cyp and 5ko lines at $3 \mathrm{hpw}$, particularly for $J A Z$ and $J A V 1$, compared to $2 a h$ that behaved like WT. This result establishes in wounded 3cyp and $5 k o$ leaves a correlation between 1/ hyperaccumulation of JA-Ile (Figure 2), 2/ absence of enhanced defense or insect resistance (Figure 3) and 3/ persistence of JAZ, JAM and JAV1 transcripts (Figure 5). In contrast, despite of enhanced JA-Ile levels upon wounding, 2ah does not display persistent 
expression of these repressors (Figure 5, see 3 and 6 hpw), and this particular pattern correlates with more robust defense (Figure 3a) and reduced insect herbivory (Figure 3b).

In response to $B$. cinerea infection, JAZ1, JAZ8 and JAM1 transcripts were overinduced in $2 a h$ and $5 k o$ (JAM2 only in 5ko) (Figure 5b), that overaccumulate JA-Ile, correlating with reduced defense (Figure 4a) and WT antifungal resistance (Figure 4b). On the contrary, these repressor genes were not overinduced in 3cyp, the only genotype exhibiting significantly increased antifungal resistance (Figure 4b). JAV1 transcripts overaccumulated in all infected mutants, but not differentially (Figure 5b). In summary, the comparative analysis across responses to two aggressors revealed that enhanced defense and resistance under deficient JAIle turnover is achieved only if negative effectors are themselves not over-responding to JAIle accumulation.

\subsection{Exogenous hormone treatments do not phenocopy genotype-specific responses of JA-} Ile catabolic mutants

Jasmonate-dependent responses are known to be largely specified by crosstalk with other hormones. Particularly, JA and ABA signaling pathways interact cooperatively in the upregulation of transcriptional wound responses whereas JA and ET act synergistically in the induction of antimicrobial defenses (Pieterse et al., 2012). We hypothetized that stressspecific responses in JA-Ile catabolic mutants could reflect different interactions of the manipulated JA pathway with ET and/or ABA pathways. To investigate this possibility, WT, $2 a h$ and 3cyp 7-days old seedlings were treated exogenously with either JA alone, or JA 
combined with the ET precursor amino-cyclopropyl-carboxylic acid (ACC), or JA combined with ABA (all $15 \mu \mathrm{M}$ ). Kinetic analysis of gene expression was conducted by RT-qPCR. All analyzed genes were found induced by JA to various extents (Figure S4), but were differentially impacted by hormone combinations. VSP1 and PDF1.2 were further stimulated by $\mathrm{JA}+\mathrm{ABA}$ and $\mathrm{JA}+\mathrm{ACC}$ treatments, respectively. As expected from known antagonism between anti-insect and antimicrobial branches of JA-defense (Pieterse et al., 2012), PDF1.2 was repressed by JA+ABA treatment, and to a lower extent, VSP1 was repressed by JA+ACC (Figure S4). Surprisingly, only very limited genotype-specific effects were recorded and here, response of defense genes was not mirrored by repressor behavior. In particular, both defense markers exhibited a stronger response in the mutants, best visualized in their respective most potent treatments, with a hyperinduction of VSP1 in 3cyp. When the three repressor gene families were analyzed, the main feature that emerged was an unexpected excessive response of 3cyp for JAZ and JAM1 genes. Remarkably, repressor responses were essentially similar to WT in 2ah for all treatments. Therefore, the hormone combinations applied produced a gene response pattern that is distinct from those arising in mutants submitted to wounding or infection stress.

\subsection{Impaired JA-Ile catabolism impacts basal JA homeostasis and differentially regulates resting levels of target transcripts}

We finally examined if impaired JA-Ile catabolism also affected basal JA homeostasis and target transcript levels in absence of imposed stress, by surveying these parameters in 
untouched leaves of a separate set of adult plants. JA profiling revealed significantly elevated JA levels in resting 3cyp and to a lesser extents in 5ko (Figure S5a). In addition, JA-Ile was clearly overaccumulated in CYP94-deficient lines, along with reduced abundance of its oxidation products. Due to high biological fluctuation in unstimulated leaves, statistical significance was difficult to ascertain for these latter, but both $12 \mathrm{OH}-\mathrm{JA}$-Ile and 12COOHJA-Ile were undetected in 3cyp as expected.

At the level of gene expression, MYC2 and ORA59 TFs transcripts were significantly upregulated in 3cyp and $5 k o$ lines (Figure S5b), but levels of their respective targets VSP and PDF1.2 were similar to or lower than WT in mutant lines. Consistently, genes encoding JAZ, $J A M$ and $J A V 1$ negative regulators had their transcripts accumulating to higher levels in 3cyp and $5 k o$, i.e. the lines with elevated JA-Ile contents. We conclude that $3 c y p$ rather than $2 a h$ restrains basal JA-Ile turnover and that non-cleared JA-Ile primarily upregulates negative feedback mechanisms, that in turn lower JA-Ile-dependent basal defenses.

\section{DISCUSSION}

Hormonal compounds must be timely and spatially controlled to ensure coordinated physiological responses. Catabolic pathways have been characterized for all major plant receptor-binding hormonal ligands and are integral components of hormone homeostasis and action. Since their recent discovery, JA-Ile catabolic pathways have been the focus of intense biochemical and physiological studies that disclosed their intricate relationships with JA signaling. As a common theme in plant hormone catabolism (Kawai, Ono, \& Mizutani, 2014; 
Mizutani \& Ohta, 2010), an oxidative inactivation pathway was characterized, with CYP94 enzymes defining a two-step JA-Ile metabolisation process. In contrast to most hormones for which conjugation corresponds to inactivation or generation of storage forms (Piotrowska \& Bajguz, 2011), JA requires conjugation to the amino acid isoleucine as the critical activation step. Consistently, deconjugation by the amidohydrolases IAR3 and ILL6 was characterized in Arabidopsis as a second JA-Ile removal pathway, acting in a JAR1 reverse reaction.

Understanding the functions of JA-Ile catabolism potentially offers avenues to tailor increased or on-demand defense signaling in diverse situations, and was addressed repetitively by lossof-function studies in the two catabolic pathways (Aubert et al., 2015; Bruckhoff et al., 2016; Heitz et al., 2012; Kitaoka et al., 2011; Koo et al., 2011; Koo et al., 2014; Luo et al., 2016; Poudel et al., 2016; Woldemariam et al., 2012; Zhang et al., 2016). However, due to the separate use of different biological systems and of partially impaired mutant lines, the possible logic underlying seemingly contradictory results was out of reach. In the present work, we conducted a comprehensive analysis of selected genotypes that allowed to evaluate the respective impact of each biochemical pathway on alterations in JA metabolism, defense regulation and resistance to biotic stress. The data clarify some former discrepancies and fill significant knowledge gaps by establishing novel pathway- and stress-specific features.

Wounding/insect stress and infection by a necrotroph both induce strong JA metabolism and signaling (Campos et al., 2014), but due to different interactions with other hormonal networks (Pieterse et al., 2012), the subsequent transcriptomes and defensive outcomes display only partial overlap. Previous reports showed that single mutations in members of 
either the oxidative or cleavage pathway were sufficient to overaccumulate JA-Ile upon leaf wounding (Heitz et al., 2012; Kitaoka et al., 2011; Koo et al., 2011; Koo et al., 2014; Widemann et al., 2013). This feature was extended in 3cyp or $2 a h$ lines fully impaired in one or the other pathway, but here we demonstrate that their simultaneous deficiency raises JA-Ile to much higher levels, indicating that upon massive and rapid JA-Ile biosynthesis triggered by mechanical wounding, no additional enzymatic pathway can efficiently turnover and limit accumulation of JA-Ile. In response to $B$. cinerea infection, a comparable JA-Ile hyperaccumulation was recorded in $2 a h$ and $5 k o$ lines, but in marked contrast 3cyp infected leaves accumulated WT levels of JA-Ile, suggesting that in this latter background, the activity of the AH pathway is sufficient to prevent abnormal hormone build-up. The CYP94 oxidation pathway is however active in infected WT plants, because oxidized JA-Ile derivatives are readily detected, albeit less abundantly than upon wounding. The metabolic preponderance of the AH pathway upon infection suggests that CYP94 cannot substitute for AH deficiency and that IAR3/ILL6 enzymes are essential for proper JA-Ile homeostasis upon necrotrophic attack. Together these results demonstrate that the two JA-Ile catabolic pathways contribute differentially to JA-Ile removal upon two distinct leaf stresses.

We next investigated the signaling output of perturbed JA-Ile catabolism in the two leaf defense models and found that transcript levels of canonical regulatory and defense markers and resistance phenotypes were overall poorly correlated with perturbed JA-Ile levels in mutant lines. Upon wounding, 3cyp was closest to WT in terms of VSP induction, contrasting with the deficient insect defense performance of this genotype. Both AH-deficient lines gained a highly persistent VSP1/2 expression, particularly marked for VSP2 in 2ah. This 
behavior was accompanied by a lower weight gain by $S$. littoralis larvae in $2 a h$, but not in $5 k o$, despite of its massive JA-Ile content. The situation with antimicrobial resistance also revealed a strong distortion of the usually linear relationship between hormone, defense and resistance levels, as the high JA-Ile-accumulating lines $2 a h$ and $5 k o$ exhibited reduced ORA59/PDF1.2 defense signaling and WT B. cinerea resistance. In contrast, 3cyp, while maintaining JA-Ile levels similar to WT, was able to better defend against the fungus, as reported earlier (Aubert et al., 2015). In this line, the behavior of the ORA59-PDF1.2 module or camalexin levels were normal and do not seem to account for the better tolerance. Nevertheless, this result shows that improved JA-dependent resistance can arise without an apparent over-accumulation of JA-Ile in a whole-leaf analysis.

The JA-Ile regulatory network is complex and sets in motion cascades of positive and negative transcriptional regulators (Hickman et al., 2017). We hypothetized that known elements defining negative feedback loops may counteract effects of chronic JA-Ile overaccumulation to restrain defense induction. This notion was introduced earlier for some $J A Z$ genes in single or double mutants of one pathway, but its relevance was difficult to assess in single biological situations investigated in a limited set of genetic backgrounds (Aubert et al., 2015; Heitz et al., 2012; Koo et al., 2011; Widemann et al., 2013). Here, we surveyed three gene families of characterized transcriptional repressors in the JA pathway in the different pathway/genotype/stress combinations: in addition to several JAZ, we also analyzed expression of $J A M$, a second type of negative regulator inhibiting transcription of JA-Ile- and MYC-regulated genes (Liu et al., 2019; Sasaki-Sekimoto et al., 2013), and JAV1, that directs 
a calcium-sensitive complex repressing JA responses (Yan et al., 2018). We found a robust negative correlation spanning both pathosystems between the behavior of several JAZ, JAM and JAV1 genes and the amplitude and timing of defense response under impaired JA-Ile turnover (summarized in Figure 6). We selected JAZ genes that were previously found to be sensitive to altered JA-Ile homeostasis (Aubert et al., 2015; Heitz et al., 2012). JAZ8, JAZ10, JAM1 and JAM2, and JAV1 expression persisted longer than in WT in 3cyp and 5ko after wounding, but strikingly behaved like WT in 2ah, correlating in this latter line with more sustained VSP expression and poorer feeding by S. littoralis larvae. In the case of $B$. cinerea infection, a similar logic was respected, but in different genotypes. Both $2 a h$ and $5 k o$ displayed significantly higher than WT expression of either JAZ or JAM or both repressor genes, but remarkably, this was not observed in 3cyp. This pattern correlated well with the former lines showing low defense and WT necrotic lesion sizes, and 3cyp maintaining WT defense and being more resistant to infection. In 3cyp, ORA59-PDF1.2 as well as camalexin accumulation behaved like in WT, so other defense determinants must account for the better resistance. In summary, we established that impairing the JA-Ile catabolic pathways has contrasted signaling consequences in Arabidopsis: a gain in defensive/resistance capacity is recorded compared to WT only when JAZ/JAM type repressor genes are not themselves overinduced, and this does not necessarily require higher hormone accumulation. In most situations, impaired JA-Ile turnover triggers a syndrome of negative feedback that likely prevents excessive signaling and that can even result in lower-than-WT defense (Poudel et al., 2016). 
The impacts of rewiring genetically JA-Ile catabolic capacity may vary between plant species and needs further investigations. For example, the oxidative or deconjugation pathway were silenced transiently (CYP94) or stably (JIH) in Nicotiana attenuata, and enhanced JA-Iledependent defenses and insect resistance were described in two studies (Luo et al., 2016; Woldemariam et al., 2012). We showed that it is possible to genetically extend the half-life and amplitude of JA-Ile pulses in Arabidopsis, but the precise mechanism underlying control of signaling when JA-Ile catabolism is impaired remains unknown. Emerging evidence indicates that distinct COI1-JAZ co-receptors have different JA-Ile detection thresholds (Chini et al., 2016; Takaoka et al., 2019) and modified JA-Ile homeostasis may selectively alter the output of some of these sensors. The mechanism directing conditional feedback hyperinduction of $J A Z / J A M$ genes is currently unknown. JA signaling is embedded in highly hierarchized hormonal networks, such as synergy with ET upon necrotroph attack, or with ABA upon wounding or herbivory, and these variable integration nodes may impact the outcome (Pieterse et al., 2012). We addressed the possible influence of these hormonal interactions by submitting young seedlings to JA treatment combined with either the ET precursor ACC or with ABA. Although a consistent but unexplained enhanced induction of both defense and repressor genes was observed in 3cyp, this hormone feeding experiment could not reproduce the genotype-specific responses arising after wounding or infection stress. Therefore, these latter cannot be explained by a simple ET vs ABA dichotomy in the integration of JA responses. 
It is remarkable that all studied mutant lines, including the newly generated $5 k o$ defective in all known JA-Ile-catabolizing enzymes, were undistinguishable from WT in their vegetative growth under standard conditions (Figure S6). This raises the question of the homeostasis of JAs and the regulation of target genes in non-stimulated leaves in these genotypes. Metabolic and molecular analysis revealed that although untouched leaves harbor a much quieter JA metabolism/signaling, a similar negative feedback operates before stress. Basal levels of stress markers are prone to fluctuations among replicates due to unintended stress during plant cultivation, but increases in steady-state JA/JA-Ile levels could be measured, particularly in 3cyp (and 5ko), and those were correlated with higher MYC2 and ORA59 transcripts (Figure S5). Hence, the action of these TFs must be somehow blocked because their target transcripts were lower than in WT, while all repressor transcripts were upregulated in mutants. These observations indicate that JA-Ile catabolism is needed on trace hormone levels to ensure optimal regulation and responsiveness to further stress. This situation is in marked contrast with the metabolic sink defined upstream of JA-Ile formation by JA oxidases (JAOs, Figure 1). The single mutation of JAO2 enhances basal JA-Ile-dependent defense and strongly increases pathogen resistance (Heitz, Smirnova, Marquis, \& Poirier, 2019; Smirnova et al., 2017).

The fact that blocking either of two catabolic pathways acting on the same JA-Ile substrate has distinct signaling and physiological outcomes suggests that signaling is not mediated solely through the control of JA-Ile steady-state levels. Modified abundance of its catabolites may also contribute to alter the signaling process(s). Recent papers reported that in addition to 
JA-Ile, 12OH-JA-Ile was an active jasmonate signaling through COI1 and contributing to wound responses (Jimenez-Aleman et al., 2019; Poudel et al., 2019). Such an interpretation would be consistent with metabolic and defensive features of $2 a h$ in wound/insect responses, but not with 3cyp in the Botrytis assay. In addition, one must keep in mind that IAR3 also accepts auxin conjugates as substrates, which may also be at the basis of hormonal cross-talk and alter signaling (Widemann et al., 2013; Zhang et al., 2016). Elucidating the factors determining when negative regulators take control will be of major importance to maximize the potential output of JA-regulated defenses. This provides the ground for future work at the protein, promoter and chromatin levels to determine how inhibitory mechanisms could be at work.

\section{Conflict of interest}

The authors declare having no conflict of interests.

\section{REFERENCES}

Aubert, Y., Widemann, E., Miesch, L., Pinot, F., \& Heitz, T. (2015). CYP94-mediated jasmonoyl-isoleucine hormone oxidation shapes jasmonate profiles and attenuates defence responses to Botrytis cinerea infection. $J$ Exp Bot, 66(13), 3879-3892. doi:10.1093/jxb/erv190. 
Browse, J. (2009). Jasmonate passes muster: a receptor and targets for the defense hormone. Annu Rev Plant Biol, 60, 183-205. doi:10.1146/annurev.arplant.043008.092007.

Bruckhoff, V., Haroth, S., Feussner, K., Konig, S., Brodhun, F., \& Feussner, I. (2016). Functional Characterization of CYP94-Genes and Identification of a Novel Jasmonate Catabolite in Flowers. PLoS One, 11(7), e0159875. doi:10.1371/journal.pone.0159875.

Campos, M. L., Kang, J. H., \& Howe, G. A. (2014). Jasmonate-triggered plant immunity. J Chem Ecol, 40(7), 657-675. doi:10.1007/s10886-014-0468-3.

Chini, A., Fonseca, S., Fernandez, G., Adie, B., Chico, J. M., Lorenzo, O., ... Solano, R. (2007). The JAZ family of repressors is the missing link in jasmonate signalling. Nature, 448(7154), 666-671. doi:10.1038/nature06006.

Chini, A., Gimenez-Ibanez, S., Goossens, A., \& Solano, R. (2016). Redundancy and specificity in jasmonate signalling. Curr Opin Plant Biol, 33, 147-156. doi:10.1016/j.pbi.2016.07.005.

Chung, H. S., Koo, A. J., Gao, X., Jayanty, S., Thines, B., Jones, A. D., \& Howe, G. A. (2008). Regulation and function of Arabidopsis JASMONATE ZIM-domain genes in response to wounding and herbivory. Plant Physiol, 146(3), 952-964. doi:10.1104/pp.107.115691.

Fernandez-Calvo, P., Chini, A., Fernandez-Barbero, G., Chico, J. M., Gimenez-Ibanez, S., Geerinck, J., ... Solano, R. (2011). The Arabidopsis bHLH transcription factors MYC3 and MYC4 are targets of JAZ repressors and act additively with MYC2 in the activation of jasmonate responses. Plant Cell, 23(2), 701-715. doi:10.1105/tpc.110.080788. 
Gidda, S. K., Miersch, O., Levitin, A., Schmidt, J., Wasternack, C., \& Varin, L. (2003). Biochemical and molecular characterization of a hydroxyjasmonate sulfotransferase from Arabidopsis thaliana. J Biol Chem, 278(20), 17895-17900. doi:10.1074/jbc.M211943200.

Glauser, G., Grata, E., Dubugnon, L., Rudaz, S., Farmer, E. E., \& Wolfender, J. L. (2008). Spatial and temporal dynamics of jasmonate synthesis and accumulation in Arabidopsis in response to wounding. $J$ Biol Chem, 283(24), 16400-16407. doi:10.1074/jbc.M801760200.

Guo, Q., Major, I. T., \& Howe, G. A. (2018). Resolution of growth-defense conflict: mechanistic insights from jasmonate signaling. Curr Opin Plant Biol, 44, 72-81. doi:10.1016/j.pbi.2018.02.009.

Havko, N. E., Major, I. T., Jewell, J. B., Attaran, E., Browse, J., \& Howe, G. A. (2016). Control of Carbon Assimilation and Partitioning by Jasmonate: An Accounting of Growth-Defense Tradeoffs. Plants (Basel), 5(1). doi:10.3390/plants5010007.

Heitz, T., Smirnova, E., Marquis, V., \& Poirier, L. (2019). Metabolic Control within the Jasmonate Biochemical Pathway. Plant Cell Physiol, 60(12), 2621-2628. doi:10.1093/pcp/pcz172.

Heitz, T., Smirnova, E., Widemann, E., Aubert, Y., Pinot, F., \& Menard, R. (2016). The Rise and Fall of Jasmonate Biological Activities. Subcell Biochem, 86, 405-426. doi:10.1007/978-3-319-25979-6_16.

Heitz, T., Widemann, E., Lugan, R., Miesch, L., Ullmann, P., Desaubry, L., ... Pinot, F. (2012). Cytochromes P450 CYP94C1 and CYP94B3 catalyze two successive oxidation 
steps of plant hormone Jasmonoyl-isoleucine for catabolic turnover. J Biol Chem, 287(9), 6296-6306. doi:10.1074/jbc.M111.316364.

Hickman, R., Van Verk, M. C., Van Dijken, A. J. H., Mendes, M. P., Vroegop-Vos, I. A., Caarls, L., ... Van Wees, S. C. M. (2017). Architecture and dynamics of the jasmonic acid gene regulatory network. Plant Cell, 29(9), 2086-2105. doi:10.1105/tpc.16.00958.

Hu, P., Zhou, W., Cheng, Z., Fan, M., Wang, L., \& Xie, D. (2013). JAV1 controls jasmonateregulated plant defense. Mol Cell, 50(4), 504-515. doi:10.1016/j.molcel.2013.04.027.

Jimenez-Aleman, G. H., Almeida-Trapp, M., Fernandez-Barbero, G., Gimenez-Ibanez, S., Reichelt, M., Vadassery, J., ... Solano, R. (2019). Omega hydroxylated JA-Ile is an endogenous bioactive jasmonate that signals through the canonical jasmonate signaling pathway. Biochim Biophys Acta Mol Cell Biol Lipids, 1864(12), 158520. doi:10.1016/j.bbalip.2019.158520.

Kawai, Y., Ono, E., \& Mizutani, M. (2014). Evolution and diversity of the 2-oxoglutaratedependent dioxygenase superfamily in plants. Plant $J, \quad 78(2), \quad 328-343$. doi:10.1111/tpj.12479.

Kitaoka, N., Matsubara, T., Sato, M., Takahashi, K., Wakuta, S., Kawaide, H., .. . Matsuura, H. (2011). Arabidopsis CYP94B3 encodes jasmonyl-L-isoleucine 12-hydroxylase, a key enzyme in the oxidative catabolism of jasmonate. Plant Cell Physiol, 52, 1757-1765. doi:10.1093/pcp/pcr110.

Koo, A. J., Cooke, T. F., \& Howe, G. A. (2011). Cytochrome P450 CYP94B3 mediates catabolism and inactivation of the plant hormone jasmonoyl-L-isoleucine. Proc Natl Acad Sci U S A, 108(22), 9298-9303. doi:10.1073/pnas.1103542108. 
Koo, A. J., \& Howe, G. A. (2012). Catabolism and deactivation of the lipid-derived hormone jasmonoyl-isoleucine. Front Plant Sci, 3, 19. doi:10.3389/fpls.2012.00019.

Koo, A. J., Thireault, C., Zemelis, S., Poudel, A. N., Zhang, T., Kitaoka, N., . . Howe, G. A. (2014). Endoplasmic reticulum-associated inactivation of the hormone jasmonoyl-Lisoleucine by multiple members of the cytochrome P450 94 family in Arabidopsis. $J$ Biol Chem, 289(43), 29728-29738. doi:10.1074/jbc.M114.603084.

Liu, Y., Du, M., Deng, L., Shen, J., Fang, M., Chen, Q., ... Zhai, Q. (2019). MYC2 Regulates the Termination of Jasmonate Signaling via an Autoregulatory Negative Feedback Loop. Plant Cell, 31(1), 106-127. doi:10.1105/tpc.18.00405.

Luo, J., Wei, K., Wang, S., Zhao, W., Ma, C., Hettenhausen, C., . . Wang, L. (2016). COI1Regulated Hydroxylation of Jasmonoyl-L-isoleucine Impairs Nicotiana attenuata's Resistance to the Generalist Herbivore Spodoptera litura. J Agric Food Chem, 64(14), 2822-2831. doi:10.1021/acs.jafc.5b06056.

Miersch, O., Neumerkel, J., Dippe, M., Stenzel, I., \& Wasternack, C. (2008). Hydroxylated jasmonates are commonly occurring metabolites of jasmonic acid and contribute to a partial switch-off in jasmonate signaling. New Phytol, 177(1), 114-127. doi:10.1111/j.1469-8137.2007.02252.x.

Mizutani, M., \& Ohta, D. (2010). Diversification of P450 genes during land plant evolution. Annu Rev Plant Biol, 61, 291-315. doi:10.1146/annurev-arplant-042809-112305.

Pieterse, C. M., Van der Does, D., Zamioudis, C., Leon-Reyes, A., \& Van Wees, S. C. (2012). Hormonal modulation of plant immunity. Annu Rev Cell Dev Biol, 28, 489-521. doi:10.1146/annurev-cellbio-092910-154055. 
Piotrowska, A., \& Bajguz, A. (2011). Conjugates of abscisic acid, brassinosteroids, ethylene, gibberellins, and jasmonates. Phytochemistry, 72(17), 2097-2112. doi:10.1016/j.phytochem.2011.08.012.

Poudel, A. N., Holtsclaw, R. E., Kimberlin, A., Sen, S., Zeng, S., Joshi, T., ... Koo, A. J. (2019). 12-Hydroxy-jasmonoyl-L-isoleucine is an active jasmonate that signals through CORONATINE INSENSITIVE 1 and contributes to the wound response in Arabidopsis. Plant Cell Physiol,. doi:10.1093/pcp/pcz109.

Poudel, A. N., Zhang, T., Kwasniewski, M., Nakabayashi, R., Saito, K., \& Koo, A. J. (2016). Mutations in jasmonoyl-L-isoleucine-12-hydroxylases suppress multiple JA-dependent wound responses in Arabidopsis thaliana. Biochim Biophys Acta, 1861(9 Pt B), 13961408. doi:10.1016/j.bbalip.2016.03.006.

Sasaki-Sekimoto, Y., Jikumaru, Y., Obayashi, T., Saito, H., Masuda, S., Kamiya, Y., . . . Shirasu, K. (2013). Basic helix-loop-helix transcription factors JASMONATEASSOCIATED MYC2-LIKE1 (JAM1), JAM2, and JAM3 are negative regulators of jasmonate responses in Arabidopsis. Plant Physiol, 163(1), 291-304. doi:10.1104/pp.113.220129.

Schweizer, F., Fernandez-Calvo, P., Zander, M., Diez-Diaz, M., Fonseca, S., Glauser, G., ... Reymond, P. (2013). Arabidopsis basic helix-loop-helix transcription factors MYC2, MYC3, and MYC4 regulate glucosinolate biosynthesis, insect performance, and feeding behavior. Plant Cell, 25(8), 3117-3132. doi:10.1105/tpc.113.115139.

Smirnova, E., Marquis, V., Poirier, L., Aubert, Y., Zumsteg, J., Menard, R., ... Heitz, T. (2017). Jasmonic Acid Oxidase 2 Hydroxylates Jasmonic Acid and Represses Basal 
Defense and Resistance Responses against Botrytis cinerea Infection. Mol Plant, 10(9), 1159-1173. doi:10.1016/j.molp.2017.07.010.

Staswick, P. E., \& Tiryaki, I. (2004). The oxylipin signal jasmonic acid is activated by an enzyme that conjugates it to isoleucine in Arabidopsis. Plant Cell, 16(8), 2117-2127. doi:10.1105/tpc.104.023549.

Takaoka, Y., Nagumo, K., Azizah, I. N., Oura, S., Iwahashi, M., Kato, N., \& Ueda, M. (2019). A comprehensive in vitro fluorescence anisotropy assay system for screening ligands of the jasmonate COI1-JAZ co-receptor in plants. J Biol Chem, 294(13), 50745081. doi:10.1074/jbc.RA118.006639.

Thines, B., Katsir, L., Melotto, M., Niu, Y., Mandaokar, A., Liu, G., . . Browse, J. (2007). JAZ repressor proteins are targets of the SCF(COI1) complex during jasmonate signalling. Nature, 448(7154), 661-665. doi:10.1038/nature05960.

Wasternack, C., \& Hause, B. (2013). Jasmonates: biosynthesis, perception, signal transduction and action in plant stress response, growth and development. An update to the 2007 review in Annals of Botany. Ann Bot, 111, 1021-1058. doi:10.1093/aob/mct067.

Wasternack, C., \& Song, S. (2017). Jasmonates: biosynthesis, metabolism, and signaling by proteins activating and repressing transcription. $J$ Exp Bot, 68(6), 1303-1321. doi:10.1093/jxb/erw443.

Widemann, E., Miesch, L., Lugan, R., Holder, E., Heinrich, C., Aubert, Y., ... Heitz, T. (2013). The amidohydrolases IAR3 and ILL6 contribute to jasmonoyl-isoleucine 
hormone turnover and generate 12-hydroxyjasmonic acid upon wounding in Arabidopsis leaves. J Biol Chem, 288(44), 31701-31714. doi:10.1074/jbc.M113.499228

Widemann, E., Smirnova, E., Aubert, Y., Miesch, L., \& Heitz, T. (2016). Dynamics of Jasmonate Metabolism upon Flowering and across Leaf Stress Responses in Arabidopsis thaliana. Plants (Basel), 5(1). doi:10.3390/plants5010004.

Woldemariam, M. G., Onkokesung, N., Baldwin, I. T., \& Galis, I. (2012). Jasmonoyl-Lisoleucine hydrolase 1 (JIH1) regulates jasmonoyl-L-isoleucine levels and attenuates plant defenses against herbivores. Plant J, 72(5), 758-767. doi:10.1111/j.1365313X.2012.05117.x.

Yan, C., Fan, M., Yang, M., Zhao, J., Zhang, W., Su, Y., ... Xie, D. (2018). Injury Activates

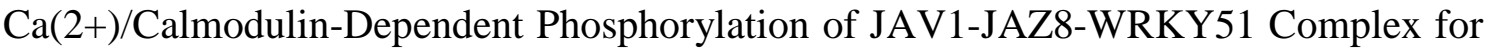
Jasmonate Biosynthesis. Mol Cell, $\quad$ 70(1), $\quad$ 136-149.e7. doi:10.1016/j.molcel.2018.03.013.

Zhang, T., Poudel, A. N., Jewell, J. B., Kitaoka, N., Staswick, P., Matsuura, H., \& Koo, A. J. (2016). Hormone crosstalk in wound stress response: wound-inducible amidohydrolases can simultaneously regulate jasmonate and auxin homeostasis in Arabidopsis thaliana. $J$ Exp Bot, 67(7), 2107-2120. doi:10.1093/jxb/erv521. 
FIGURE 1. Positions of the impaired enzymatic steps in JA-Ile catabolic mutants and simplified view of analyzed signaling and defense responses. Left box: Necrotrophic fungus infection or mechanical wounding/insect attack trigger jasmonic acid (JA) biosynthesis. Some JA is conjugated to isoleucine by JAR1 enzyme to form bioactive jasmonoyl-isoleucine (JAIle). JA-Ile is turned over by a two-step oxidation to $12 \mathrm{OH}-\mathrm{JA}-\mathrm{Ile}$ and $12 \mathrm{COOH}-\mathrm{JA}-\mathrm{Ile}$ by the cytochrome P450 enzymes CYP94B3/B1 and CYP94C1, or by conjugate cleavage mediated by the amidohydrolases (AH) IAR3 and ILL6. These AH also cleave 12OH-JA-Ile to release 12OH-JA. Red crosses indicate impaired JA-Ile catabolic steps in higher order mutants utilized in this study: oxidation-deficient (triple cyp94b1 b3 c1 mutant, 3cyp), deconjugationdeficient (double iar3 ill6, 2ah) or deficient for both pathways (quintuple mutant, 5ko). Right box: in absence of JA-Ile, JAZ, JAM and JAV1 repressors block transcription of target genes. W51: WRKY51. Upon biosynthesis, JA-Ile promotes the assembly of the COI1-JAZ coreceptor which is recruited into the $\mathrm{SCF}^{\mathrm{COI} 1} \mathrm{E} 3$ ubiquitin ligase directing proteolytic degradation of JAZ repressors. Transcription factors (TFs) like MYC2 or ORA59 become activated in association with mediator 25 subunit (MED25) and allow the transcription of numerous stimulus-specific defense responses. The anti-insect branch was probed by the MYC2-VSP genes, and the antimicrobial branch by the ORA59 and PDF1.2 genes. Among JA-Ile targets are also genes encoding JAZ repressors, JAM proteins that compete with MYC2 and ORA59, to attenuate signaling. In mutant plants impaired for JA-Ile catabolism, overinduction of this negative feedback loop may occur and prevent hyper-stimulation of JAIle-dependent defenses and associated enhanced resistance.

FIGURE 2. JA-Ile and oxidized catabolites accumulation in 2ah, 3cyp and $5 k o$ mutants after 
mechanical wounding or Botrytis cinerea infection. Six-week-old plants were wounded 3 times across leaf mid-vein (a) or drop-inoculated on two sites per leaf with a suspension containing $2.5 \times 10^{6}$ fungal spores $\mathrm{mL}^{-1}$ (b). Treated leaves were harvested at 1,3 and 6 hours post wounding (hpw) or 3 days post inoculation (dpi) on WT (black bars), 2ah (yellow bars), 3cyp (blue bars) and 5ko (green bars) plants. Jasmonates were extracted and quantified by LC-MS/MS. JA-Ile, 12OH-JA-Ile and 12COOH-JA-Ile levels were expressed in nmol g-1 fresh weight $(\mathrm{FW})$. Histograms represent the mean \pm SEM of three (wounding) or four (B. cinerea) biological replicates. Columns labeled with different letters indicate a significant difference between genotypes at each time point as determined by one-way ANOVA with Tukey post-hoc test, $\mathrm{P}<0.05$.

FIGURE 3. Expression profiles of JA-Ile-dependent genes in response to mechanical wounding (a) and impact on insect feeding (b) in JA-Ile catabolic mutants. Expression profiles of jasmonate-dependent MYC2, VSP1 and VSP2 marker genes were determined in response to wounding in WT (black bars), 2ah (yellow bars), 3cyp (blue bars) and 5ko (green bars) mutants. Relative expression of each target gene at 1, 3 or 6 hours post wounding (hpw) is represented. Gene expression was determined by real-time PCR using gene-specific primers and normalized using EXP and TIP41 reference genes. Transcript quantification was performed on three biological replicates analyzed in duplicate. Histograms represent mean expression \pm SEM. Columns labeled with different letters indicate a significant difference between genotypes at each time point as determined by one-way ANOVA with Tukey posthoc test, $\mathrm{P}<0.05$. For insect feeding assay, S. littoralis larvae were placed on leaves of 4 week-old plants. After 8-9 days, larval weight was determined. Histograms represent mean \pm 
SEM from 3 independent trials (presented in Figure S3). The number of total larvae on each genotype is indicated within the bars. Different letters indicate significant differences at $P$ $<0.05$ (linear mixed model).

FIGURE 4. Expression profiles (a) of JA-Ile-dependent defense genes and (b) resistance levels in response to $B$. cinerea infection in 2ah, 3cyp and 5ko mutants. (a) Expression profiles of jasmonate-regulated ORA59 and PDF1.2 genes in response to infection by $B$. cinerea in WT (black bars), 2ah (yellow bars), 3cyp (blue bars) and 5ko (green bars) mutants. Relative expression of each target gene at 3 day post infection (dpi) is represented. Gene expression was determined by real-time PCR using gene-specific primers and normalized using EXP and TIP41 reference genes. Transcript quantification was performed on three biological replicates analyzed in duplicate. Histograms represent mean expression \pm SEM. Columns labeled with different letters indicate a significant difference between genotypes at each time point as determined by one-way ANOVA with Tukey posthoc test, $\mathrm{P}<0.05$. (b) For disease resistance assessment, two sites per leaf were inoculated across the main vein with $5 \mu \mathrm{L}$ of spore suspension containing $2.5 \times 10^{6}$ spores $\mathrm{mL}^{-1}$. Representative leaves of each genotype were detached and photographed at 3 dpi (left panel). Histograms represent the mean lesion diameters \pm SEM of about 100 lesion sites from 10 to 15 plants for each genotype (middle panel). Fungal growth was evaluated by real-time qPCR with $B$. cinerea cutinase-specific primers on genomic DNA extracted from 3-day- infected leaves (right panel). Quantification was performed on three biological replicates analyzed in duplicate. Columns labeled with different letters indicate a significant difference as determined by oneway ANOVA with Tukey post-hoc test $(\mathrm{P}<0.01)$. 
FIGURE 5. Expression profiles of JA-Ile dependant repressor genes in response to wounding and infection in 2ah, 3cyp and 5ko mutants in response to wounding (a), or infection by Botrytis cinerea (b), in WT (black bars), 2ah (yellow bars), 3cyp (blue bars) and 5ko (green bars) mutants. Relative expression of each target gene at 1, 3 or 6 hours post wounding (hpw) and 3 day post infection (dpi) is represented. Gene expression was determined by real-time PCR using gene-specific primers and normalized using EXP and TIP41 reference genes. Transcript quantification was performed on three biological replicates analyzed in duplicate. Histograms represent mean expression \pm SEM. Columns labeled with different letters indicate a significant difference between genotypes at each time point as determined by one-way ANOVA with Tukey posthoc test, $\mathrm{P}<0.05$.

FIGURE 6. Relationships between impaired JA-Ile catabolism, JA-Ile levels, defense/repressor gene induction, and resistance to attackers. The correlations show distinct circuitry for the two leaf responses analyzed. The signs -, 0 , or + indicate lower, equal, or increased response respectively, in stimulated mutant genotypes compared to WT. The two catabolic pathways have differential contributions to JA-Ile turnover in response to wounding or infection. JA-Ile accumulation is enhanced to different extents, except in infected 3cyp, and this has variable consequences on defense amplitude and resistance to aggressors. The trend emerging is that impaired hormone catabolism does, with or without JA-Ile overaccumulation, result in better defense/resistance, only if negative effectors like $J A Z$ or $J A M$ are themselves not overinduced. 
FIGURE 1

\section{Stimulus}

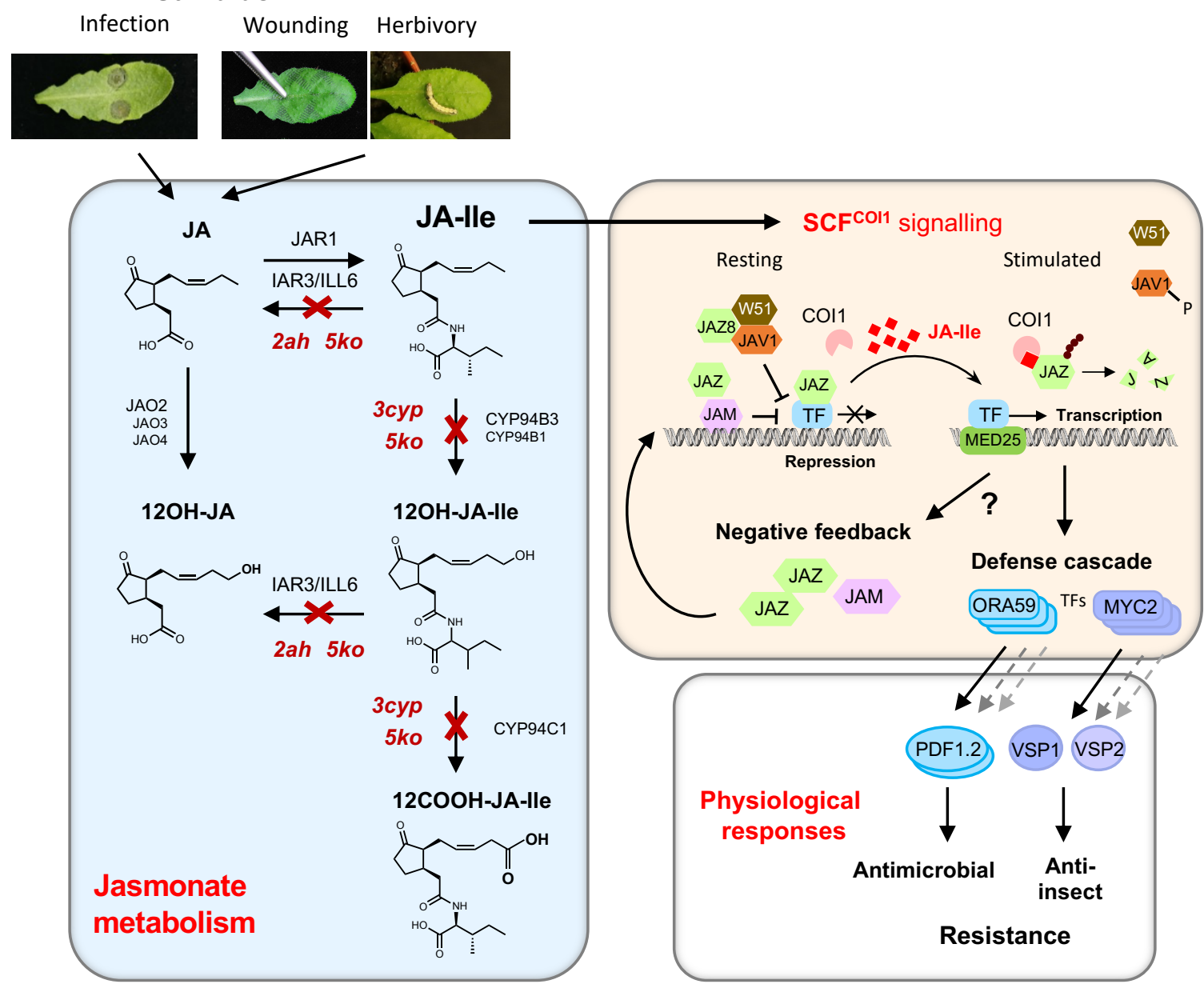


FIGURE 2.

(a)
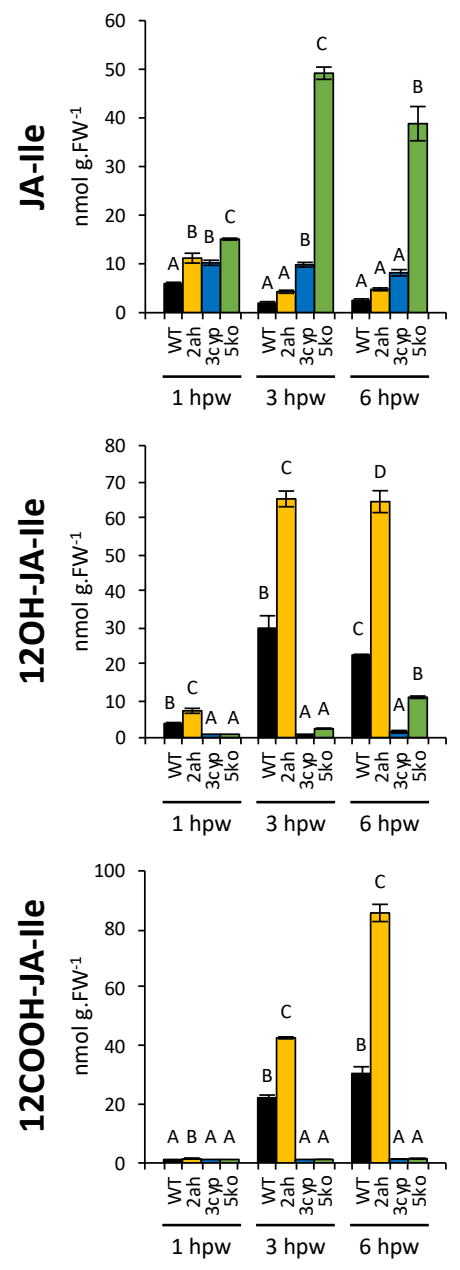

(b)
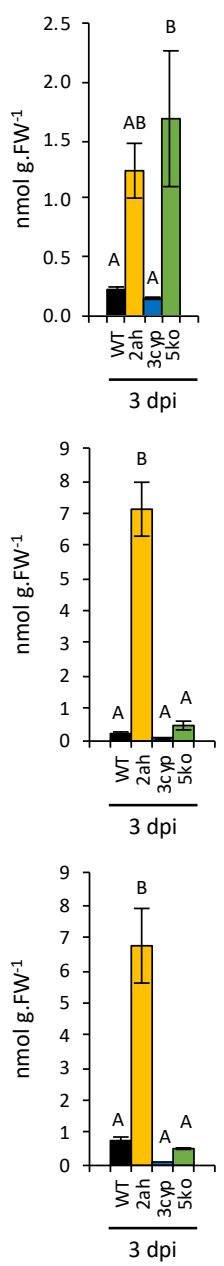


\section{FIGURE 3.}

(a)

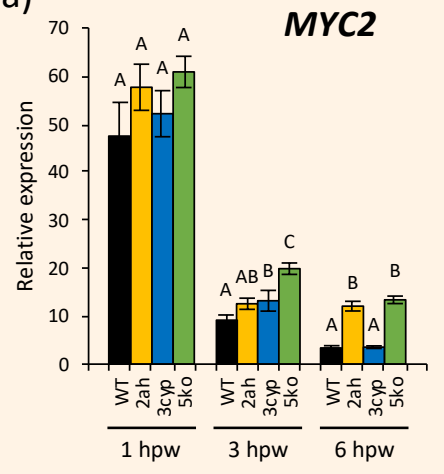

(b)

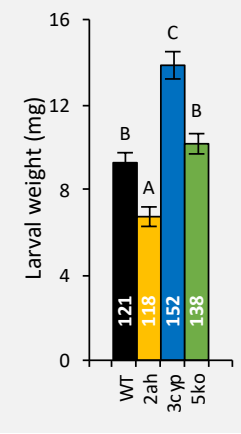

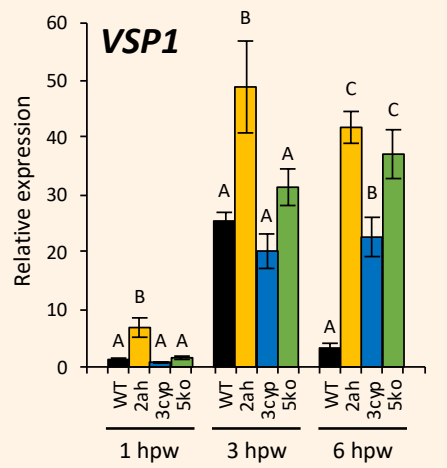

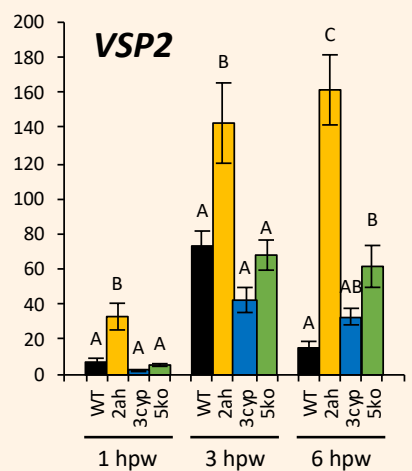


FIGURE 4.

$\begin{array}{lll}\text { (a) ORA59 PDF1.2 (b) } & \end{array}$

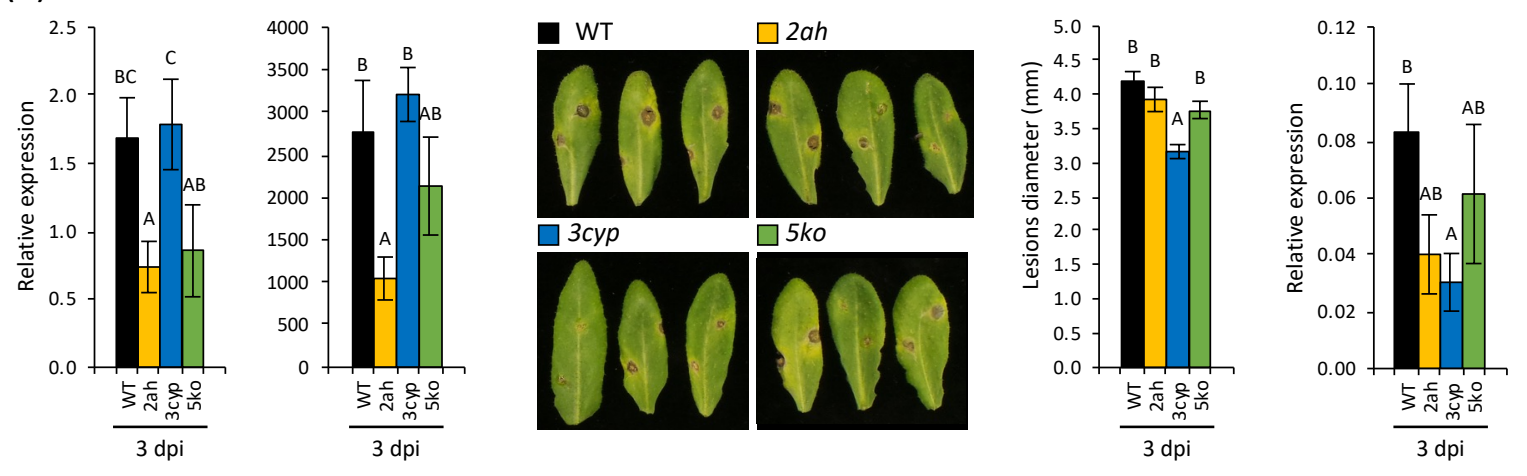


FIGURE 5

(a)

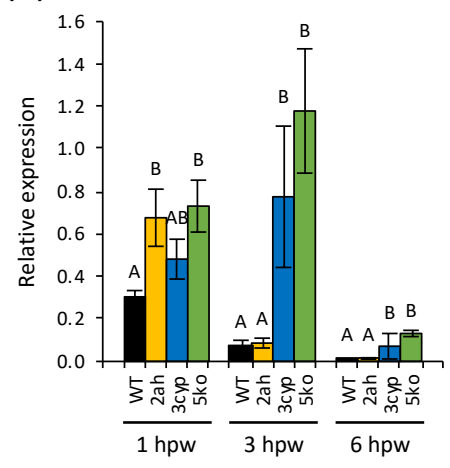

JAM1
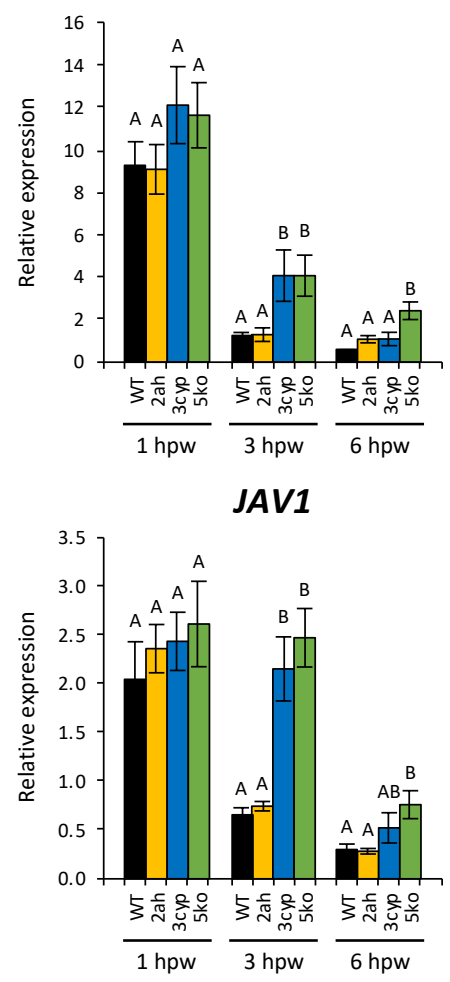

JAZ10
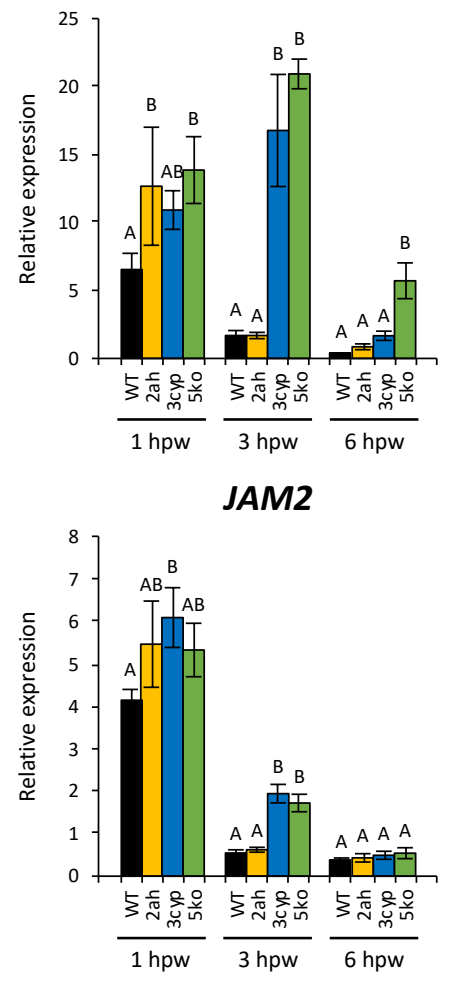

(b)
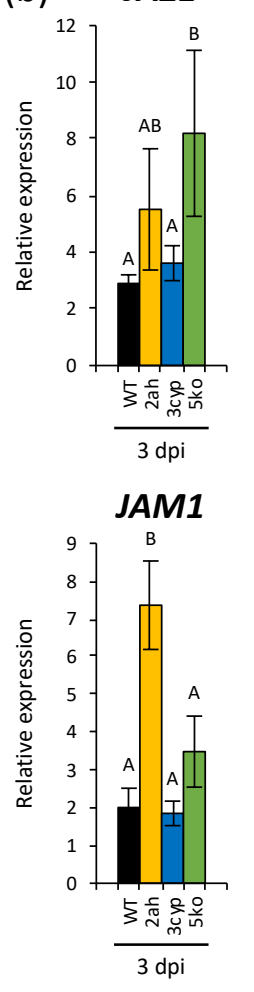

JAV1

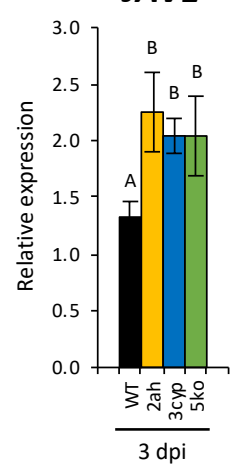

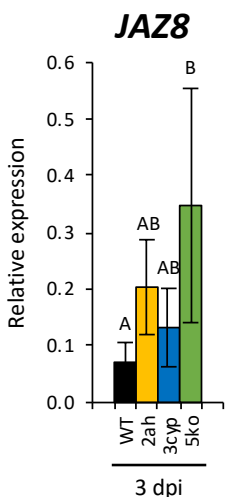

JAM2

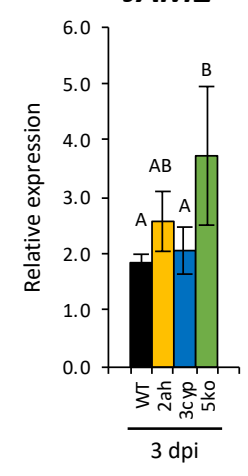




\section{FIGURE 6}

\begin{tabular}{|c|c|c|c|c|c|}
\hline \multicolumn{7}{|c|}{ Wound/Insect } \\
genotype & $\begin{array}{c}\text { Impaired } \\
\text { pathway }\end{array}$ & JA-lle levels & $\begin{array}{c}\text { Defense } \\
\text { transcript } \\
\text { induction }\end{array}$ & $\begin{array}{c}\text { Repressor } \\
\text { transcript } \\
\text { induction }\end{array}$ & $\begin{array}{c}\text { Resistance } \\
\text { phenotype }\end{array}$ \\
\hline $3 c y p$ & oxidation & + & 0 & ++ & -- \\
\hline $2 a h$ & deconjugation & + & ++ & 0 & + \\
\hline $5 k o$ & both & +++ & + & ++ & 0 \\
\hline
\end{tabular}

\begin{tabular}{|c|c|c|c|c|c|}
\hline \multicolumn{7}{|c|}{ B. cinerea infection } \\
gutant & $\begin{array}{c}\text { Impaired } \\
\text { pathway }\end{array}$ & JA-Ile levels & $\begin{array}{c}\text { Defense } \\
\text { transcript } \\
\text { induction }\end{array}$ & $\begin{array}{c}\text { Repressor } \\
\text { transcript } \\
\text { induction }\end{array}$ & $\begin{array}{c}\text { Resistance } \\
\text { phenotype }\end{array}$ \\
\hline $3 c y p$ & oxidation & 0 & 0 & 0 & + \\
\hline $2 a h$ & deconjugation & ++ & - & + & $0 /+$ \\
\hline $5 k o$ & both & ++ & - & + & 0 \\
\hline
\end{tabular}


FIGURE S1

Marquis et al.

(a)

IAR3

ILL6
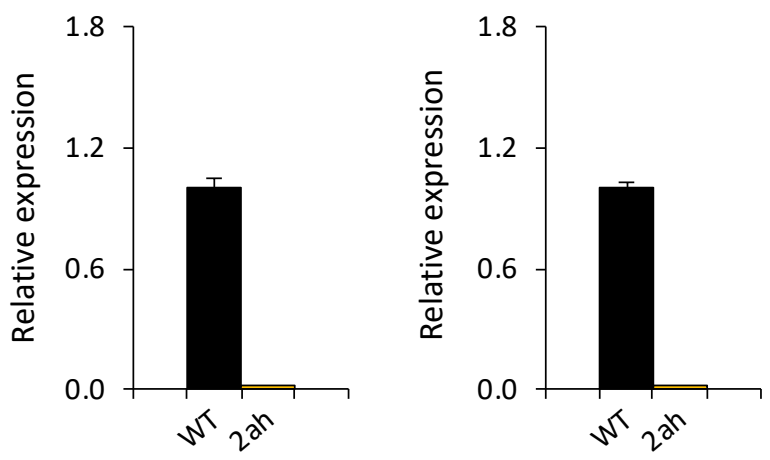

(b) CYP94B1
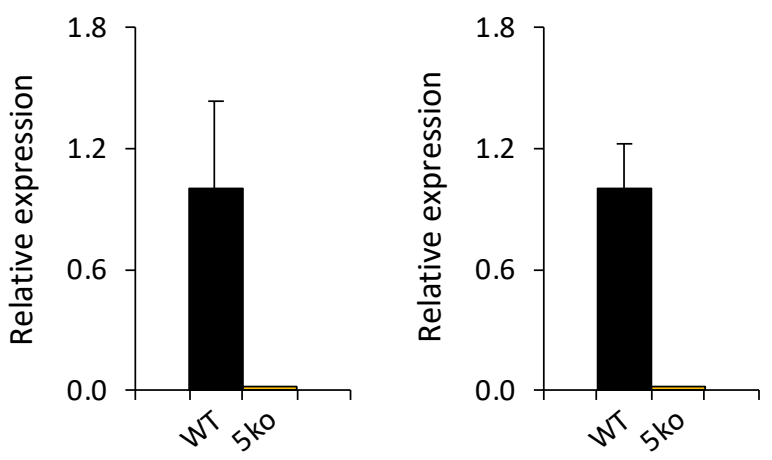

CYP94C1

IAR3

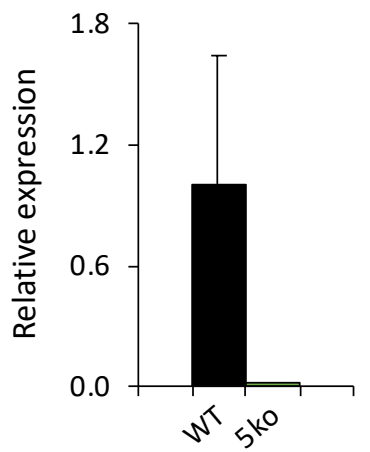

ILL6

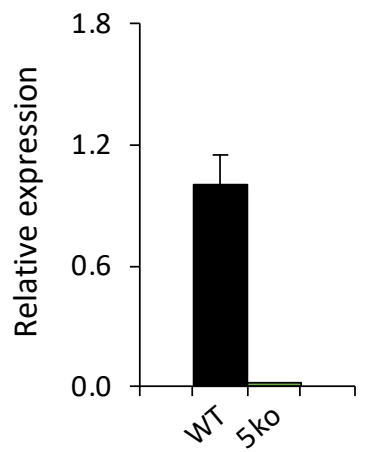


(a)

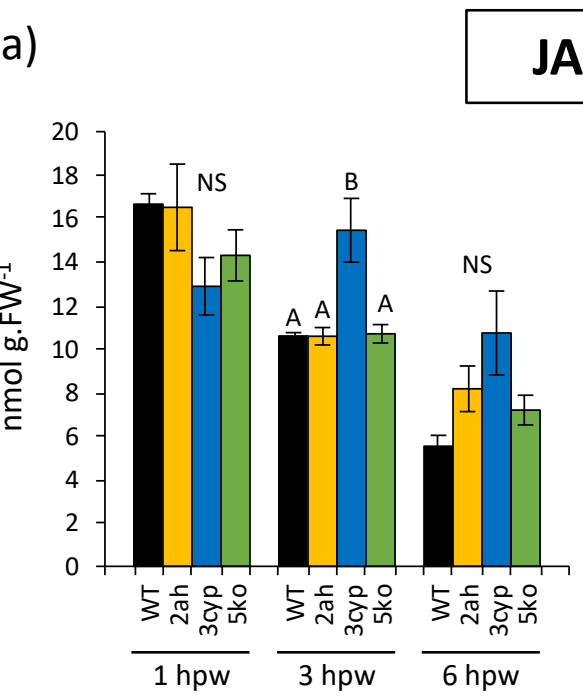

(c)

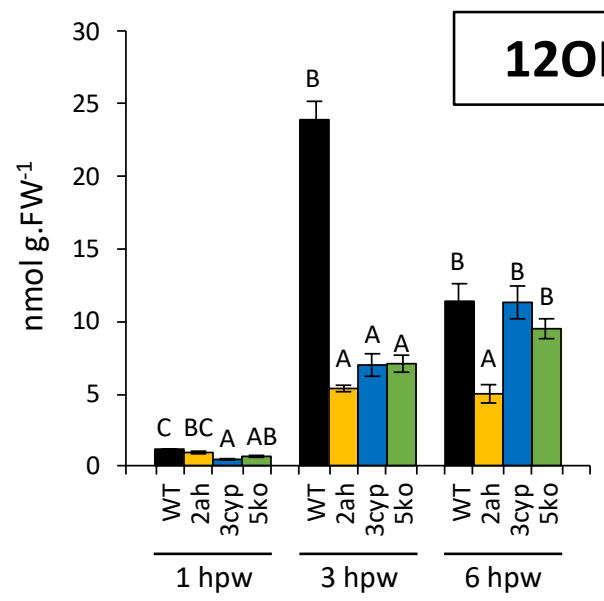

(b)

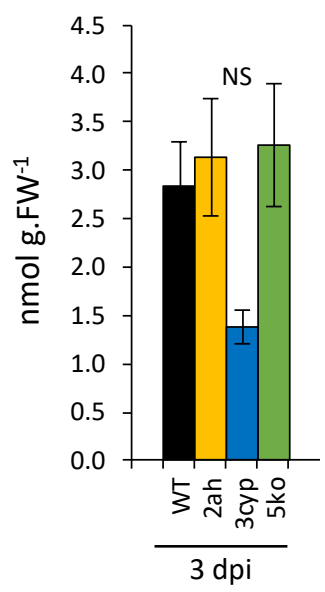

(d)

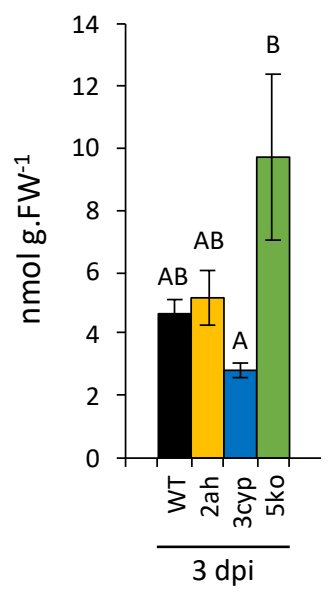


FIGURE S3

Trial 1

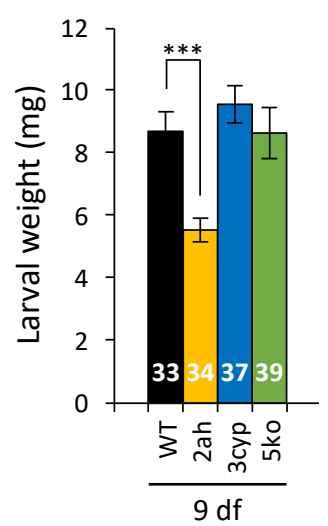

Trial 2

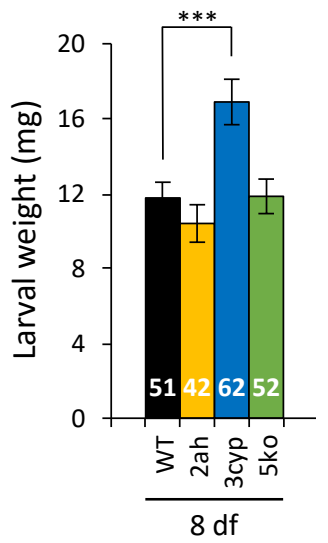

Marquis et al.

Trial 3

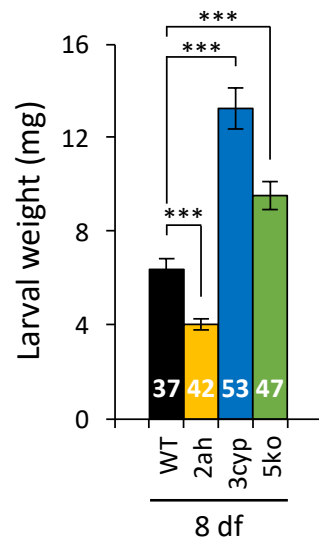


FIGURE S4.

Marquis et al.

Mock
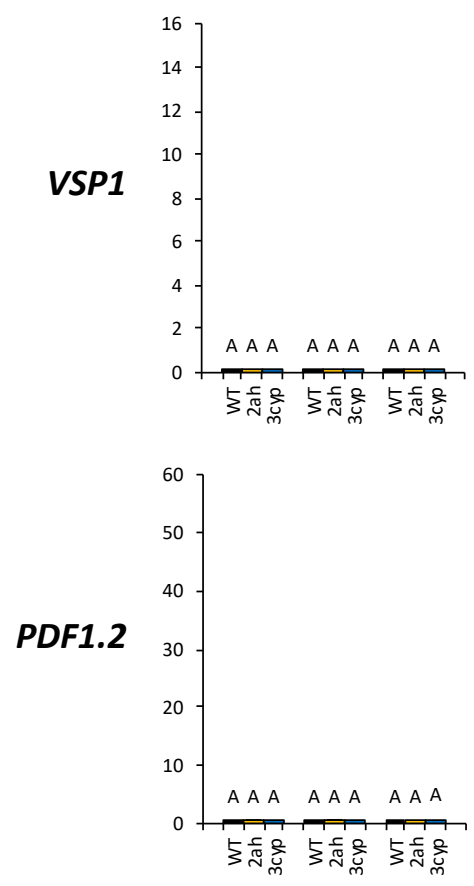

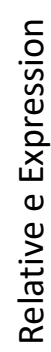
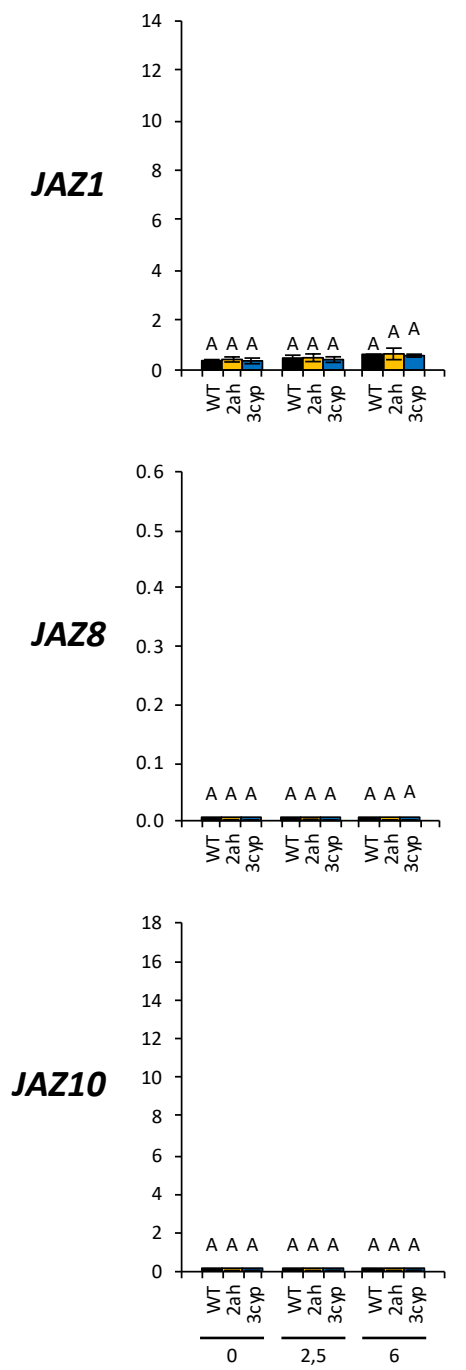

JA
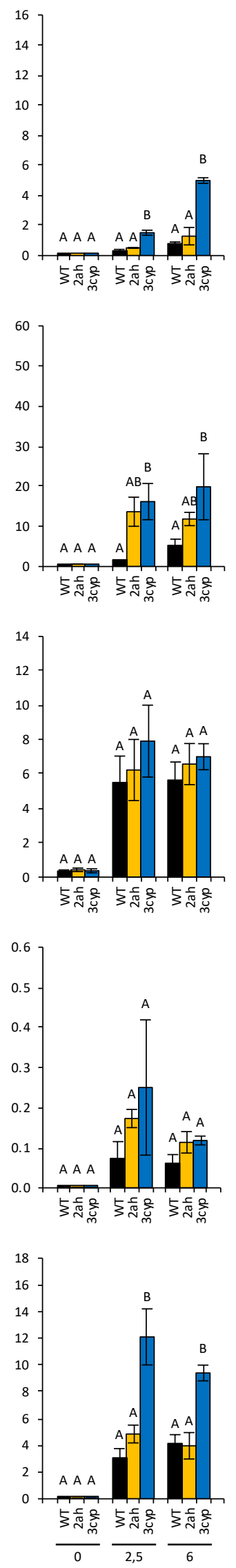

JA + ACC
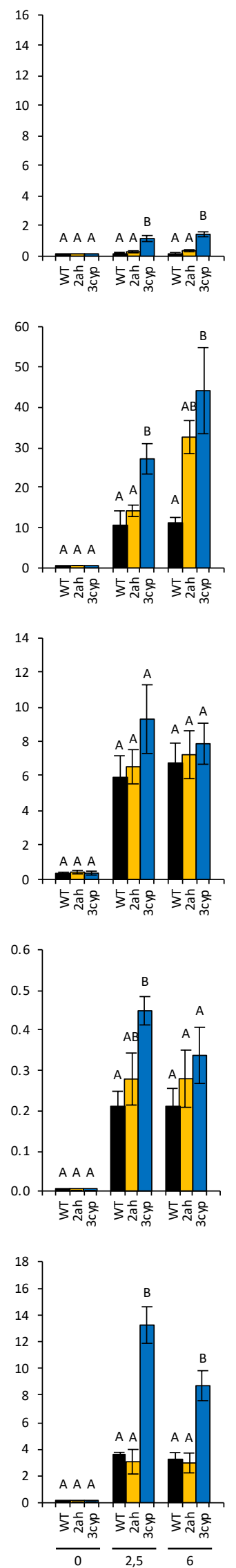

JA + ABA
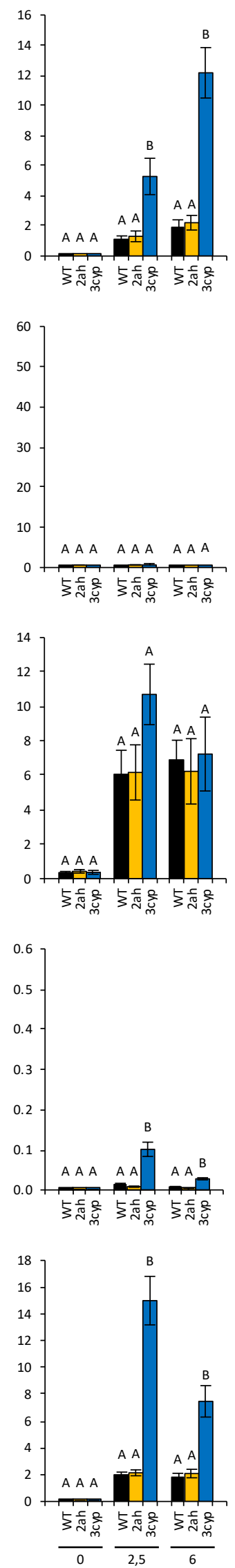

Hours post-treatment 

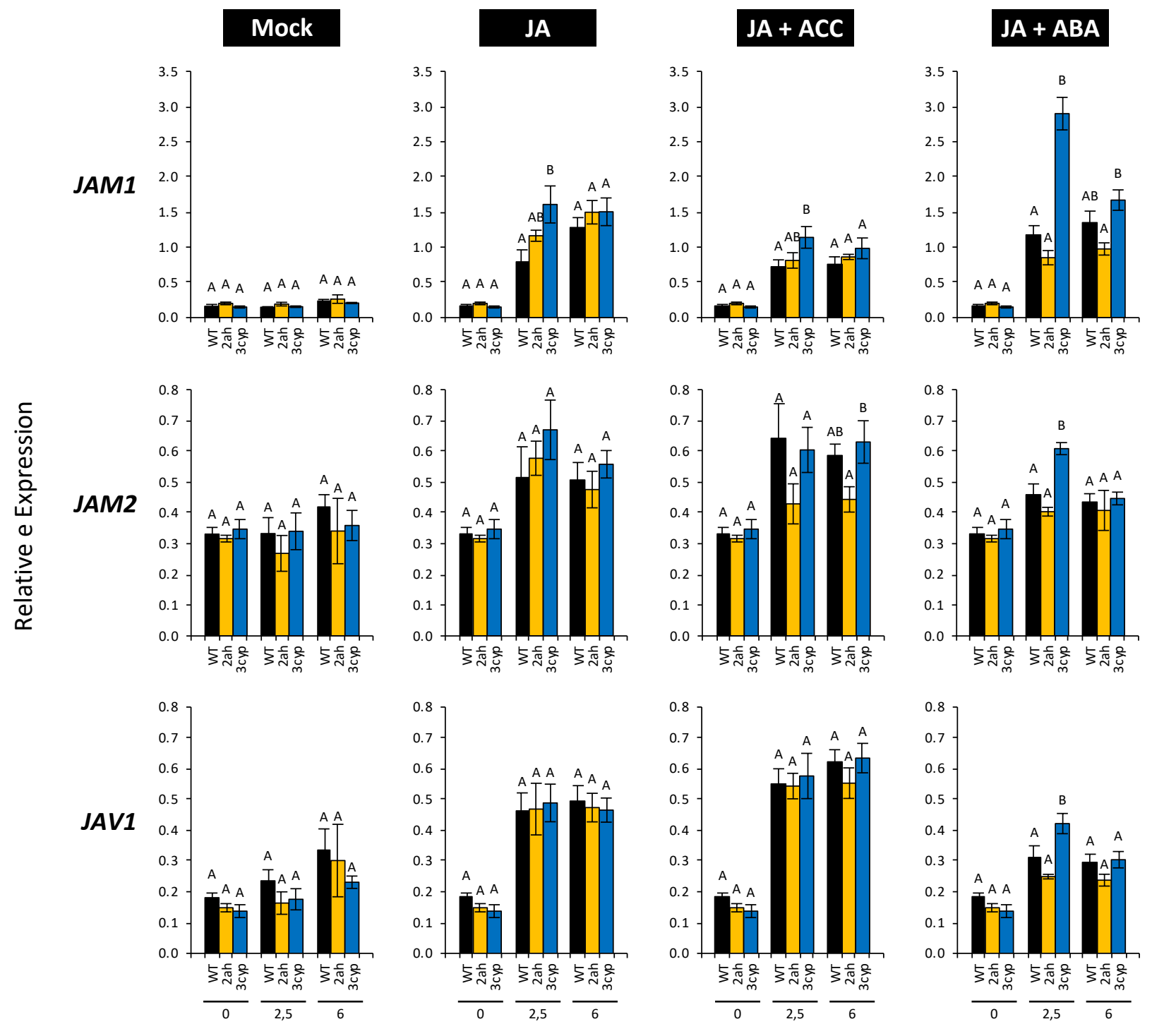

Hours post-treatment 

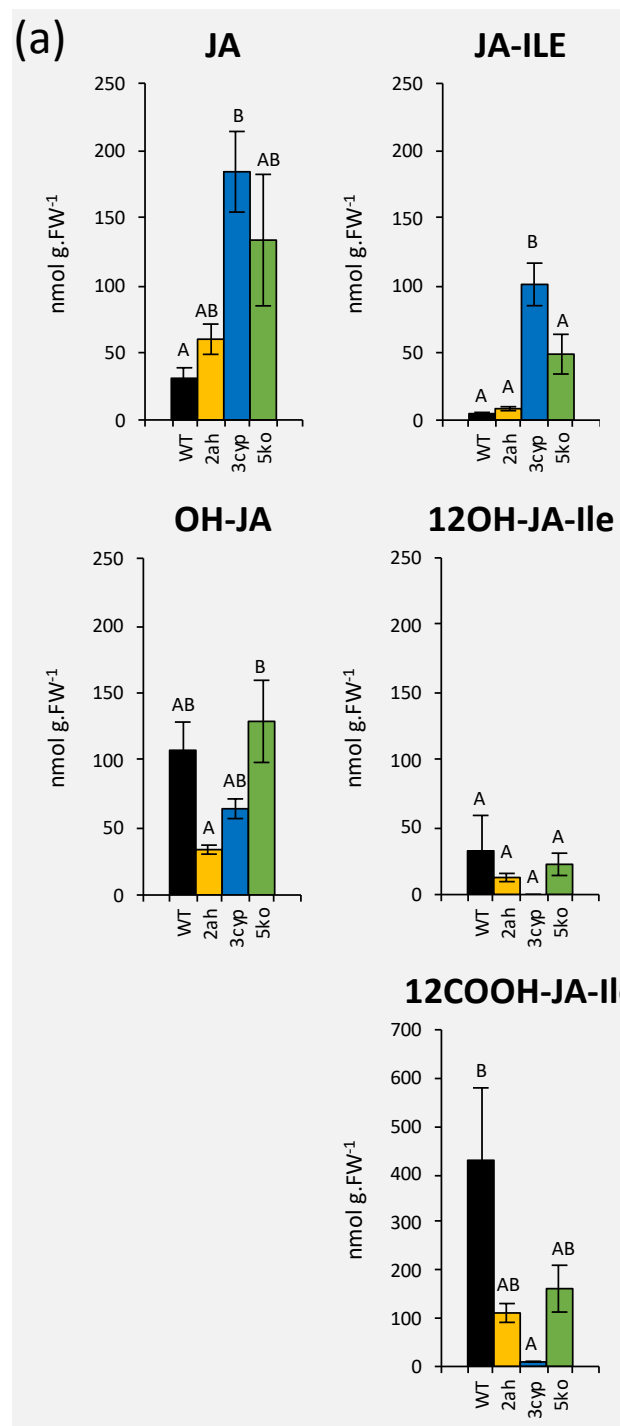

(b)

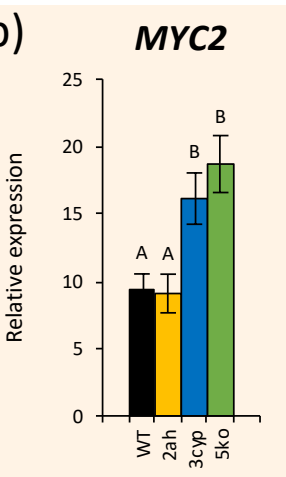

ORA59

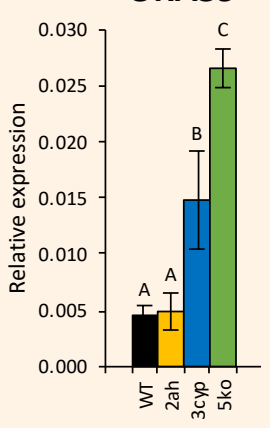

JAZ1
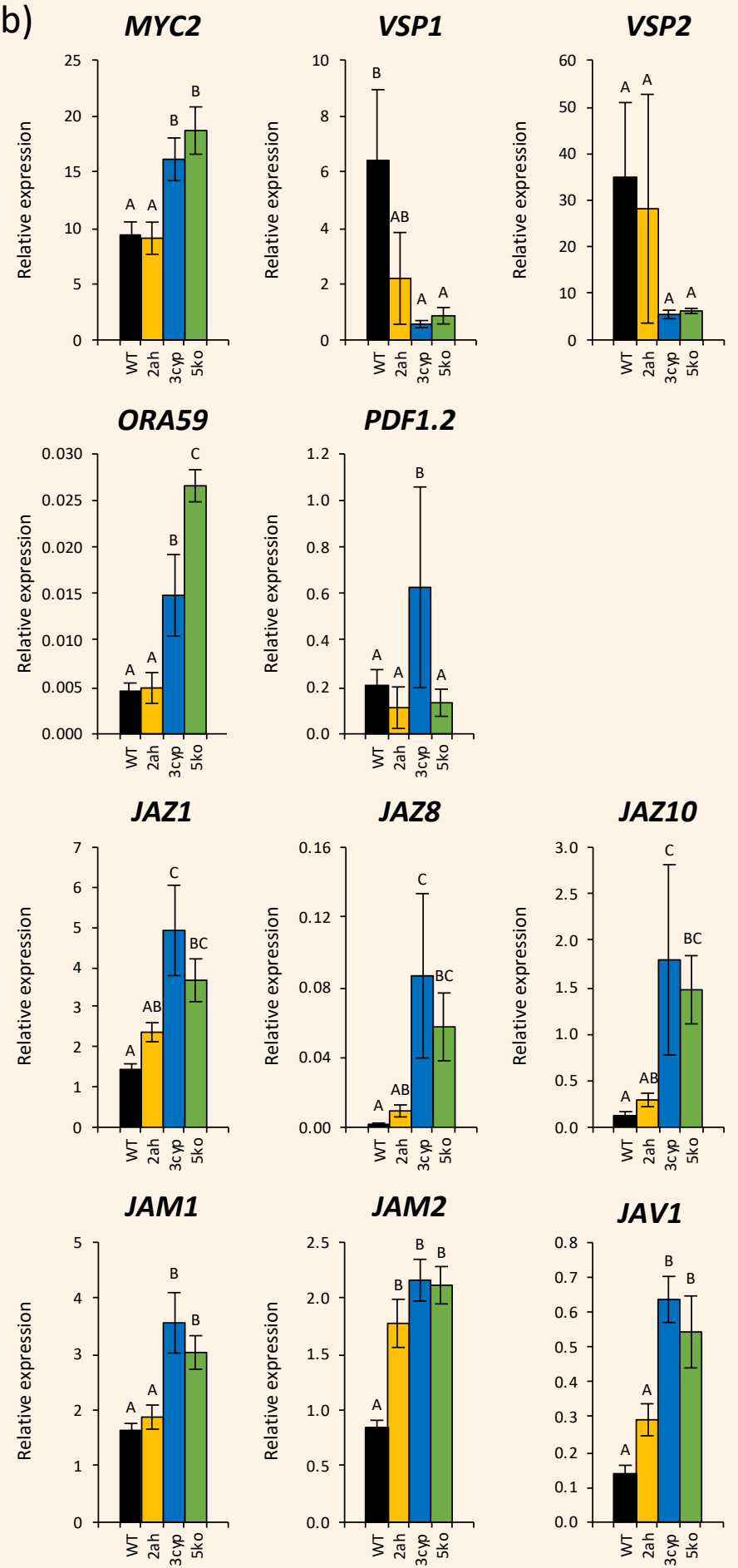

PDF1.2
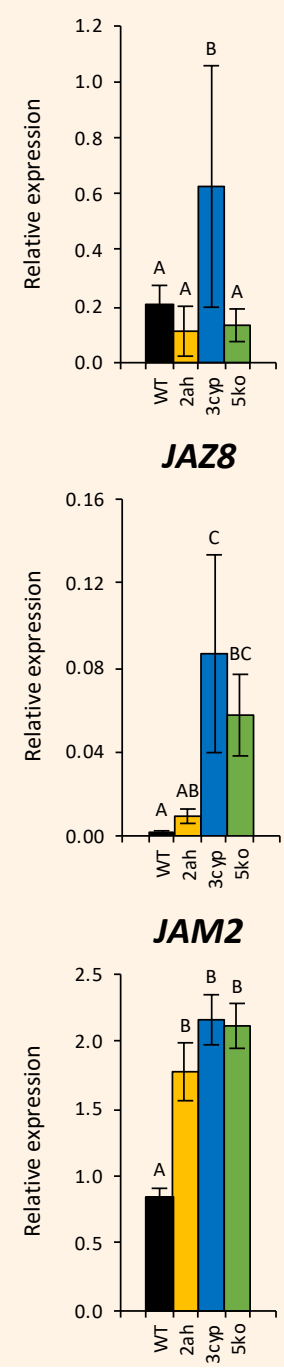

JAV1

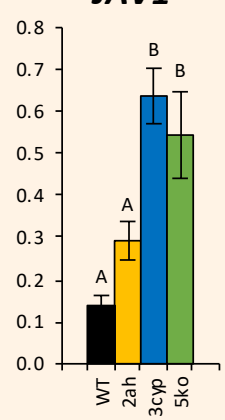




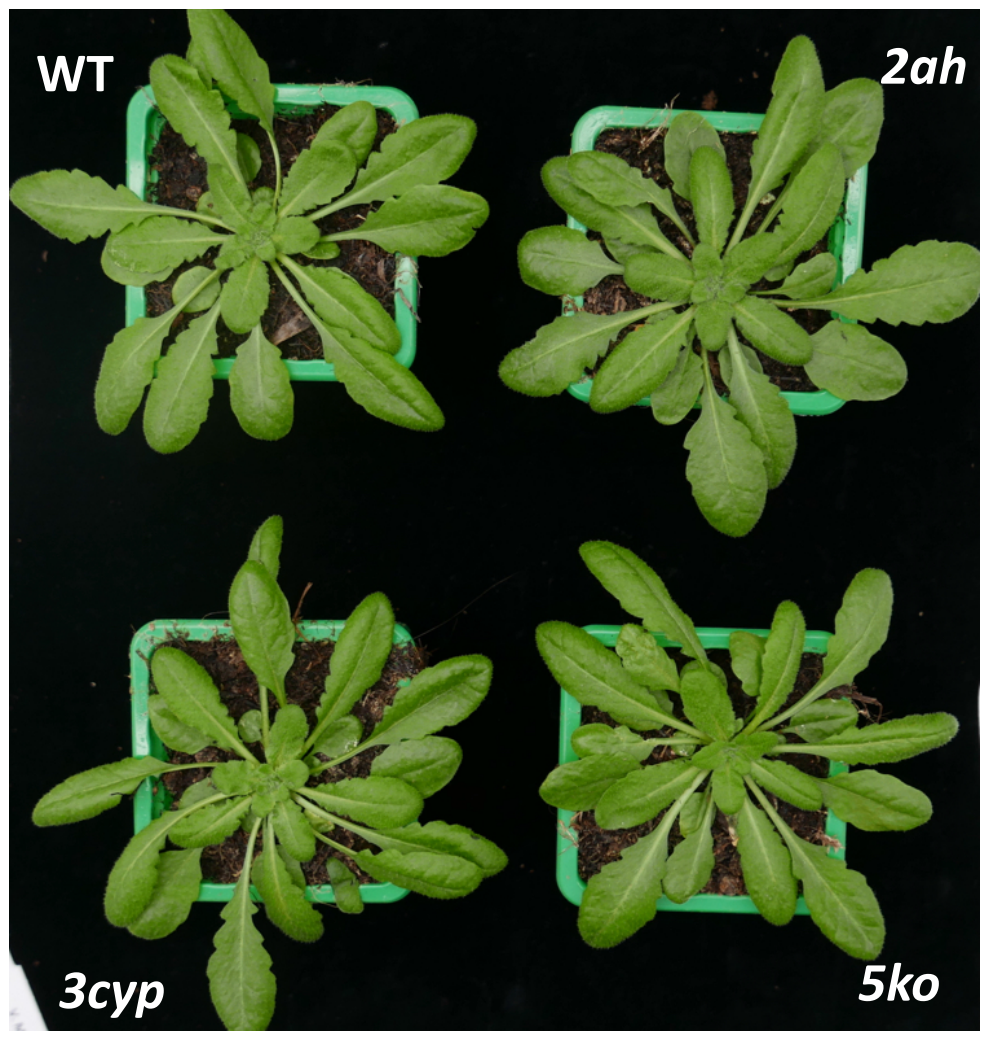




\begin{tabular}{|c|c|c|c|}
\hline \multicolumn{4}{|l|}{ qPCR } \\
\hline Gene/allele & Locus & Primer name & Sequence $\left(5^{\prime}->3^{\prime}\right)$ \\
\hline \multirow{2}{*}{$E X P$} & At4g26410 & At4g26410-gPCR F & gagctgaagtggcttcaatgac \\
\hline & & At4g26410-qPCR R & ggtccgacatacccatgatcc \\
\hline \multirow[t]{2}{*}{ TIP4.1 } & At4g34270 & TIP41 LP & gtgaaaactgttggagagaagcaa \\
\hline & & TIP41 RP & tcaactggataccctttcgca \\
\hline \multirow[t]{2}{*}{ MYC2 } & At1g32640 & E49-MYC2-L & gccgaaggaatacacgcaat \\
\hline & & E50-MYC2-R & cgggttgtgaacgggcta \\
\hline \multirow[t]{2}{*}{ VSP1 } & At5g24780 & VSP1-F & ccgtcaatgtttggatctttg \\
\hline & & VSP1-R & gctgtgttctcggtcccata \\
\hline \multirow[t]{2}{*}{ VSP2 } & At5g24770 & VSP2_qPCR for & ggtgcccgcaaattgcaaagacta \\
\hline & & VSP2_gPCR rev & ggttgatgctccggtccctaacca \\
\hline \multirow[t]{2}{*}{ ORA59 } & At1g06160 & ORA59_qPCR for_new & ttcgacgttgacatcttctcc \\
\hline & & ORA59_qPCR rev new & tcttgcgtcataacaacactctg \\
\hline \multirow[t]{2}{*}{ PDF1.2 } & At5g44420 & E39-PDF1.2-L & cacccttatcttcgctgctctt \\
\hline & & E40-PDF1.2-R & tacacttgtgtgctgggaagac \\
\hline \multirow[t]{2}{*}{$J A Z 1$} & At1g19180 & JAZ1-F & ttctgagttcgtcggtagcc \\
\hline & & JAZ1-R & cacgtctgtgagaagctaggc \\
\hline \multirow[t]{2}{*}{ JAZ8 } & At1g30135 & JAZ8-F & aatgtgttttcttcagatgttaccc \\
\hline & & JAZ8-R & ttctctgcttgcgatcgatatt \\
\hline \multirow[t]{2}{*}{ JAZ10 } & At5g13220 & JAZ10-F & cgctcctaagcctaagttcca \\
\hline & & JAZ10-R & tttcgaaatcgcaccttgaat \\
\hline \multirow[t]{2}{*}{ JAM1 } & At2g46510 & JAM1_qPCR for & ggagctcacgcgtatcctaa \\
\hline & & JAM1_qPCR_rev & ggattcgaagaagcagcaac \\
\hline \multirow[t]{2}{*}{ JAM2 } & At1g01260 & JAM2_qPCR for & gaggccaatcaacgtgaagg \\
\hline & & JAM2_qPCR_rev & tcccctcctccttctccatc \\
\hline \multirow[t]{2}{*}{ CYP94B1 } & At5g63450 & CYP94B1 qPCR F62 & caatgaggctttacccaccag \\
\hline & & CYP94B1 qPCR R62 & aaatgtcgtcgtttgctgcat \\
\hline \multirow{2}{*}{ CYP94B3 } & At3g48520 & CYP94B3 qPCR F62 & tggettacacgaaggcttgtc \\
\hline & & CYP94B3 qPCR R62 & agtcccacgaaactggaggat \\
\hline \multirow[t]{2}{*}{ CYP94C1 } & At2g27690 & CYP94C1 qPCR F63 & ggcccggattacgaagagttt \\
\hline & & CYP94C1 qPCR R63 & ggccggaacttaccttcgtt \\
\hline \multirow[t]{2}{*}{ IAR3 } & At1g51760 & IAR3-0,4F & gttgctttaagggctgatatgg \\
\hline & & IAR3-N69047-R & accgagaagcatcgtagtgtga \\
\hline \multirow[t]{2}{*}{ ILL6 } & At1g44350 & ILL6-GK212E12-F & gtgtcccatatccatccaacgg \\
\hline & & ILL6-GK212E12-R & agactaatgaccgcggaagaag \\
\hline Actin2 & At3g18780 & Act_for & cttgcaccaagcagcatgaa \\
\hline & & Act rev & ccgatccagacactgtacttcctt \\
\hline UBQ10 & At4g05320 & UBQ10 for & ggccttgtataatccctgatgaataag \\
\hline & & UBQ10 rev & aaagagataacaggaacggaaacatagt \\
\hline Cutinase & B.c Z69264 & CutA-L & gatgtgacggtcatcttgccc \\
\hline & & CutA-R & agatttgagagcggcgagg \\
\hline T-DNA genot & ping & & \\
\hline Gene/allele & & Primer name & Sequence $\left(5^{\prime} \rightarrow>3^{\prime}\right)$ \\
\hline & & LBb1.3 (SALK) & attttgccgatttcggaac \\
\hline & & GABI8409LB & atattgaccatcatactcattgc \\
\hline cyp94b1-1 & & 94B1-LP & tcgaatcacattgctctctcc \\
\hline & & 94B1-RP & gggaattcactttcgaaatcc \\
\hline cyp94b3-1 & & CYP94B3-0,72F & gaacgtgggaagcgagaggaagc \\
\hline & & BO1BG68 & tggtttggttctcactgttcac \\
\hline сур94c1-1 & & SALK_055455 LP & tgtctttttggaaagtagcacc \\
\hline & & SALK_055455 RP & gattccacggcctaaaagatc \\
\hline iar3-5 iar3-7 & & Salk_069047-LP & gttctccacgtgcgttatagc \\
\hline & & Salk_069047-RP & aaaaagccacactgttccatg \\
\hline ill6-1 & & GK412E11-LP & gactatgcttcttggtgctgc \\
\hline & & GK412E11-RP & cgcacctcttgaatacgtttc \\
\hline ill6-2 & & SALK_024894-LP & gactatgcttcttggtgctgc \\
\hline & & SALK_024894-RP & cgcacctcttgaatacgtttc \\
\hline
\end{tabular}

